\title{
On the Calabi problem: a finite-dimensional approach
}

Received November 23, 2010 and in revised form August 8, 2011

\begin{abstract}
Let us consider a projective manifold $M^{n}$ and a smooth volume form $\Omega$ on $M$. We define the gradient flow associated to the problem of $\Omega$-balanced metrics in the quantum formalism, the $\Omega$-balancing flow. We prove that at the limit of the quantization, the $\Omega$-balancing flow converges towards a natural flow in Kähler geometry, the $\Omega$-Kähler flow. We also prove the long time existence of the $\Omega$-Kähler flow and its convergence towards Yau's solution to the Calabi problem of prescribing the volume form in a given Kähler class (see Theorem 2). We derive some natural geometric consequences of our study.
\end{abstract}

\section{The $\Omega$-balancing flow}

In this first section we give some definitions and recall some natural moment map considerations relating to $\Omega$-balanced metrics. Then we introduce the two main flows of this paper, the $\Omega$-balancing flow and the $\Omega$-Kähler flow, and state our main results, Theorems 1 and 2.

Assume that $M$ is a smooth polarized manifold of complex dimension $n$, and $L$ an ample line bundle. We consider a smooth volume form $\Omega$ on $M$ such that $\int_{M} \Omega=$ $\operatorname{Vol}_{L}(M):=c_{1}(L)^{n}$, the volume of $M$ with respect to $L$.

In [Don09], S. K. Donaldson introduced the notion of $\Omega$-balanced metric, adapted to the Calabi problem of fixing the volume of a Kähler metric in a given Kähler class. These metrics are algebraic metrics coming from Kodaira's embedding of the manifold in $\mathbb{P} H^{0}\left(L^{k}\right)^{\vee}$ for $k$ sufficiently large. More precisely, given a (smooth) hermitian metric $h \in \operatorname{Met}\left(L^{k}\right)$, one can consider the Hilbertian map

$$
\operatorname{Hilb}_{\Omega}=\operatorname{Hilb}_{k, \Omega}: \operatorname{Met}\left(L^{k}\right) \rightarrow \operatorname{Met}\left(H^{0}\left(L^{k}\right)\right)
$$

such that

$$
\operatorname{Hilb}_{\Omega}(h)=\int_{M} h(\cdot, \cdot) \Omega
$$

H.-D. Cao: Department of Mathematics, University of Macau, Macau, China, and Department of Mathematics, Lehigh University, Bethlehem, PA 18015 USA; e-mail: huc2@lehigh.edu

J. Keller: Centre de Mathématiques et Informatique, Université d'Aix-Marseille, Technopole Château-Gombert, 39, rue F. Joliot Curie, 13453 Marseille Cedex 13, France;

e-mail: jkeller@cmi.univ-mrs.fr 
is the $L^{2}$ metric induced by the fibrewise $h$ and the volume form $\Omega$. On the other hand, one can consider the Fubini-Study maps

$$
\mathrm{FS}=\mathrm{FS}_{k}: \operatorname{Met}\left(H^{0}\left(L^{k}\right)\right) \rightarrow \operatorname{Met}\left(L^{k}\right)
$$

such that for $H \in \operatorname{Met}\left(H^{0}\left(L^{k}\right)\right),\left\{S_{i}\right\}$ an $H$-orthonormal basis of $H^{0}\left(L^{k}\right)$ and for all $p \in M$,

$$
\sum_{i=1}^{\operatorname{dim} H^{0}\left(L^{k}\right)}\left|S_{i}(p)\right|_{\mathrm{FS}(H)}^{2}=\frac{\operatorname{dim} H^{0}\left(L^{k}\right)}{\operatorname{Vol}_{L}(M)},
$$

thus fixing pointwise the metric $\operatorname{FS}(H) \in \operatorname{Met}\left(L^{k}\right)$. One of the main results of [Don09] asserts that the dynamical system

$$
T_{k}=\mathrm{FS} \circ \mathrm{Hilb}_{\Omega}
$$

has a unique attractive fixed point.

Definition 1.1. Let $(M, L)$ be a polarized manifold, and $\Omega$ a smooth volume form. Then for any sufficiently large $k$, there exists a unique fixed point $h_{k}$ of the map $T_{k}: \operatorname{Met}\left(L^{k}\right)$ $\rightarrow \operatorname{Met}\left(L^{k}\right)$ which is called $\Omega$-balanced. The metric $\operatorname{Hilb}_{\Omega}\left(h_{k}\right) \in \operatorname{Met}\left(H^{0}\left(L^{k}\right)\right)$ and the Kähler form $c_{1}\left(h_{k}\right) \in 2 \pi c_{1}(L)$, given by the curvature of $h_{k}$, will also be called $\Omega$-balanced.

When $k$ tends to infinity, one obtains from [Don09] and [Kel09, Theorem 3] the following result.

Theorem 1.1. As $k \rightarrow \infty$, the sequence of normalized $\Omega$-balanced metrics $h_{k}^{1 / k} \in$ $\operatorname{Met}(L)$ converges to a hermitian metric $h_{\infty}$ in smooth topology and its curvature is a solution to the Calabi problem of prescribing the volume form ${ }^{1}$ in a given Kähler class,

$$
c_{1}\left(h_{\infty}\right)^{n}=\Omega \text {. }
$$

Let us denote $N+1=N_{k}+1=\operatorname{dim} H^{0}\left(L^{k}\right)$. Another way of presenting the notion of $\Omega$-balanced metric is to introduce a moment map setting. Let us consider first $\mu: \mathbb{C P}^{N} \rightarrow$ $i \mathfrak{u}(N+1)$ which is a moment map for the $U(N+1)$ action and the Fubini-Study metric $\omega_{\mathrm{FS}}$ on $\mathbb{C P}^{N}$. Note that here we identify implicitly the Lie algebra $\mathfrak{u}(N+1)$ with its dual using the bilinear form $(A, B)=-\operatorname{tr}(A B)$. Given homogeneous unitary coordinates, one sets explicitly $\mu=(\mu)_{\alpha, \beta}$ as

$$
\left(\mu\left(\left[z_{0}, \ldots, z_{N}\right]\right)\right)_{\alpha, \beta}=\frac{z_{\alpha} z_{\beta}}{\sum_{i}\left|z_{i}\right|^{2}}
$$

Then, given a holomorphic embedding $\iota: M \hookrightarrow \mathbb{P} H^{0}\left(L^{k}\right)^{\vee}$, we can consider the integral of $\mu$ over $M$ with respect to the volume form

$$
\mu_{\Omega}(\iota)=\int_{M} \mu(\iota(p)) \Omega(p),
$$

\footnotetext{
1 Note that in the rest of the paper we shall ignore the normalization factor $1 / n$ ! in front of the Monge-Ampère mass $c_{1}\left(h_{\infty}\right)^{n}$.
} 
which induces a moment map for the $\mathrm{U}(N+1)$ action over the space of all bases of $H^{0}\left(L^{k}\right)$.

Let us give some details on that point. On the space $\mathfrak{M}$ of smooth maps from $M$ to $\mathbb{P} H^{0}\left(L^{k}\right)^{\vee}$, we have a natural symplectic structure $\varpi$ defined by

$$
\varpi(a, b)=\int_{M}(a, b) \Omega
$$

for $a, b \in T_{\iota} \mathfrak{M}$ and $(\cdot, \cdot)$ the Fubini-Study inner product induced on the tangent vectors. Let $\zeta \in \mathfrak{u}(N+1)$ and $X_{\zeta} \in H^{0}\left(\mathbb{P}^{N \vee}, T \mathbb{P}^{N \vee}\right)$ be the induced holomorphic vector field on $\mathbb{P}^{N \vee}=\mathbb{P} H^{0}\left(L^{k}\right)^{\vee}$. For all $Y \in \Gamma\left(M, T \mathbb{P}_{\mid M}^{N \vee}\right)$ we have

$$
\begin{aligned}
\varpi\left(X_{\zeta \mid M}, Y\right) & =\int_{M} i_{Y}\left(i_{X_{\zeta}} \omega_{\mathrm{FS}}\right) \Omega=-\int_{M} \operatorname{tr}(d \mu(Y) \cdot \zeta) \Omega \\
& =-\operatorname{tr}\left(d \mu_{\Omega}(Y) \cdot \zeta\right)=\left(d \mu_{\Omega}(Y), \zeta\right)
\end{aligned}
$$

and $\mu_{\Omega}$ is Ad-equivariant as the integral of the Ad-equivariant moment map $\mu$. Thus, $\mathrm{U}(N+1)$ acts isometrically on $\mathfrak{M}$ with the moment map given by

$$
\iota \mapsto-\sqrt{-1}\left(\mu_{\Omega}(\iota)-\frac{\operatorname{tr}\left(\mu_{\Omega}(\iota)\right)}{N+1} \operatorname{Id}_{N+1}\right) \in i \mathfrak{s u}(N+1) .
$$

Note that if one defines a hermitian metric $H$ on $H^{0}\left(L^{k}\right)$, one can consider an orthonormal basis with respect to $H$ and the associated embedding, and thus it also makes sense to speak of $\mu_{\Omega}(H)$. As we shall see, in the Bergman space $\mathcal{B}=\mathcal{B}_{k}=\operatorname{GL}(N+1) / \mathrm{U}(N+1)$, we have a preferred metric associated to the volume form $\Omega$ and the moment map we have just defined, and this is precisely an $\Omega$-balanced metric.

Definition 1.2. The embedding $\iota$ is $\Omega$-balanced if

$$
\mu_{\Omega}^{0}(\iota):=\mu_{\Omega}(\iota)-\frac{\operatorname{tr}\left(\mu_{\Omega}(\iota)\right)}{N+1} \operatorname{Id}_{N+1}=0 .
$$

An $\Omega$-balanced embedding corresponds (up to $\mathrm{SU}(N+1)$-isomorphisms) to an $\Omega$-balanced metric $\iota^{*} \omega_{\mathrm{FS}}$ by pull-back of the Fubini-Study metric from $\mathbb{P} H^{0}\left(L^{k}\right)^{\vee}$, so our two definitions actually coincide (see [Don09]). Note that for $H \in \operatorname{Met}\left(H^{0}\left(L^{k}\right)\right)$, it also makes sense to consider $\mu_{\Omega}(h)$ where $h=\operatorname{FS}(H) \in \operatorname{Met}\left(L^{k}\right)$, i.e. when $h$ belongs to the space of Bergman type fibrewise metrics that we identify with $\mathcal{B}$.

On the other hand, seen as a hermitian matrix, $\mu_{\Omega}^{0}(\iota)$ induces a vector field on $\mathbb{C P}^{N}$. Thus, inspired from [Fin10], we study the flow

$$
\frac{d \iota(t)}{d t}=-\mu_{\Omega}^{0}(\iota(t))
$$

which we call the $\Omega$-balancing flow. To fix the starting point of this flow, we choose a Kähler metric $\omega=\omega(0)$ and we construct a sequence of hermitian metrics $h_{k}(0)$ such that $\omega_{k}(0):=c_{1}\left(h_{k}(0)\right)$ converges smoothly to $\omega(0)$ providing a sequence of embeddings 
$\iota_{k}(0)$ for $k \gg 0$. Such a sequence of embeddings is known to exist thanks to Theorem 2.1. For technical reasons, we decide to rescale this flow by considering the ODE

$$
\frac{d \iota_{k}(t)}{d t}=-k \mu_{\Omega}^{0}\left(\iota_{k}(t)\right)
$$

which we call the rescaled $\Omega$-balancing flow. Of course, we are interested in the behaviour of the sequence of Kähler metrics $\omega_{k}(t)=(1 / k) \iota_{k}(t)^{*}\left(\omega_{\mathrm{FS}}\right)$ when $t$ and $k$ tend to infinity. Here is one of the main results of this paper.

Theorem 1. For any fixed $t$, the sequence $\omega_{k}(t)$ converges in $C^{\infty}$ topology to the solution $\omega+\sqrt{-1} \partial \bar{\partial} \phi_{t}$ of the Monge-Ampère equation

$$
\frac{\partial \phi_{t}}{\partial t}=1-\frac{\Omega}{\left(\omega+\sqrt{-1} \partial \bar{\partial} \phi_{t}\right)^{n}}
$$

with $\phi_{0}=0$ and $\omega=\lim _{k \rightarrow \infty} \omega_{k}(0)$. Furthermore, the convergence is $C^{1}$ in the variable $t$.

We call the flow given by (3) the $\Omega$-Kähler flow. The proof of this theorem will be done in several steps. First we study the limit of a convergent sequence of rescaled $\Omega$-balancing flows to identify the limit (Section 2), which we shall call the $\Omega$-Kähler flow. Then, in Section 3, we study in detail the behaviour of the $\Omega$-Kähler flow in any Kähler class and prove our second main result.

Theorem 2. Let $\phi_{t}$ be the solution to (3) on the maximal time interval $0 \leq t<T_{\max }$. Let

$$
v_{t}=\phi_{t}-\frac{1}{\operatorname{Vol}_{L}(M)} \int_{M} \phi_{t} \frac{\omega^{n}}{n !} .
$$

Then the $C^{\infty}$ norm of $v_{t}$ is uniformly bounded for all $0 \leq t<T_{\max }$ and consequently $T_{\max }=+\infty$. Moreover, as $t \rightarrow \infty$, $v_{t}$ converges to $v_{\infty}$ in smooth topology and $\partial \phi_{t} / \partial t$ converges to a constant in smooth topology.

Finally, inspired by [Don01] and especially [Fin10] for the Calabi flow, we will prove Theorem 1 in Section 4. In Section 5, we give a moment map interpretation of the $\Omega$ Kähler flow and draw some possible generalizations.

\section{The limit of the rescaled $\Omega$-balancing flow}

In this section, we assume that the sequence $\omega_{k}(t)$ is convergent and we want to relate its limit to (3). The goal of this section is to prove the following result.

Theorem 3. Suppose that for each $t \in \mathbb{R}_{+}$, the metric $\omega_{k}(t)$ induced by (2) converges in smooth topology to a metric $\omega_{t}$ and that this convergence is $C^{1}$ in $t \in \mathbb{R}_{+}$. Then the limit $\omega_{t}$ is a solution to the flow (3) starting at $\omega_{0}=\lim _{k \rightarrow \infty} \omega_{k}(0)$. 
Given a matrix $H$ in $\operatorname{Met}\left(H^{0}\left(L^{k}\right)\right)$, we obtain a vector field $X_{H}$ which induces a perturbation of any embedding $\iota: M \hookrightarrow \mathbb{P} H^{0}\left(L^{k}\right)^{\vee}$. The induced infinitesimal change in $\iota^{*} \omega_{\mathrm{FS}}$ is pointwise given by the potential $\operatorname{tr}(H \mu)$ where $\mu$ is given by (1). Thus, the corresponding potential in the case of the rescaled $\Omega$-balancing flow is

$$
-k \operatorname{tr}\left(\mu_{\Omega}^{0} \mu\right) \text {. }
$$

Since we are rescaling the flow in (2) and considering forms in the class $2 \pi c_{1}(L)$, we are led to understand the asymptotic behaviour as $k \rightarrow \infty$ of the potentials

$$
\beta_{k}=-\operatorname{tr}\left(\mu_{\Omega}^{0} \mu\right)
$$

We will need the following key result. Let us fix a Kähler form $\omega \in 2 \pi c_{1}(L)$ and write $\omega=c_{1}(h)$. Let us define a different Hilbertian map

$$
\text { Hilb : } \operatorname{Met}\left(L^{k}\right) \rightarrow \operatorname{Met}\left(H^{0}\left(L^{k}\right)\right)
$$

by setting

$$
\operatorname{Hilb}(h)=\int_{M} h(\cdot, \cdot) \omega^{n}=\int_{M} h(\cdot, \cdot) c_{1}(h)^{n}
$$

Theorem 2.1 (Asymptotic expansion of the Bergman kernel). The Bergman function associated to $h^{k}$ has the pointwise asymptotic expansion

$$
\rho_{k}(h)(p):=\sum_{i=1}^{N+1}\left|s_{i}\right|_{h^{k}}^{2}(p)=k^{n}+\sum_{i \geq 1} k^{n-i} a_{i}(h),
$$

where $\left(s_{i}\right)_{i=1}^{N+1}$ is a Hilb $\left(h^{k}\right)$-orthonormal basis of $H^{0}\left(L^{k}\right)$. Here the $a_{i}(h)$ depend on the curvature and its covariant derivatives and are uniformly bounded on $M$. If $h$ is varying in a compact set (in smooth topology) in the space of hermitian metrics with positive curvature, then

$$
\left\|\rho_{k}(h)-k^{n}+\sum_{i=1}^{m} k^{n-i} a_{i}(h)\right\|_{C^{r}} \leq \frac{C}{k^{m+1}},
$$

where $C$ is uniform and only depends on $r$.

$A$ direct consequence is the convergence of the sequence of Bergman metrics $(1 / k) c_{1}\left(\mathrm{FS}\left(\operatorname{Hilb}\left(h^{k}\right)\right)\right)$ to $\omega$ in smooth topology, i.e. for all $r \geq 0$,

$$
\left\|\frac{1}{k} c_{1}\left(\mathrm{FS}\left(\operatorname{Hilb}\left(h^{k}\right)\right)\right)-\omega\right\|_{C^{r}}=O(1 / k) .
$$

Theorem 2.1 is nowadays usually called the Tian-Yau-Zelditch expansion. S.-T. Yau conjectured the convergence of the Bergman metrics in [Yau86, Section 6.1], while G. Tian proved it in [Tia90] for $C^{2}$ topology (and Y.-D. Ruan for $C^{\infty}$, see [Rua98]) and identified $a_{0}=1$. The existence of the asymptotic expansion was proved S. Zelditch [Ze198] (and independently by D. Catlin [Cat99]) using Boutet de Monvel-Sjöstrand techniques. The uniformity of the coefficients $a_{i}$ appeared in [Lu00] and will be crucial in the rest of the paper. We refer to [MM07] for a general survey on this topic, which also provides a historical perspective. 
Remark 2.1. The function $\rho_{k}(h)$ is the restriction to the diagonal of the kernel of the orthogonal $L^{2}$-projection (with respect to $h$ and $\operatorname{Hilb}\left(h^{k}\right)$ ) from the space of smooth sections of $L^{k}$ to the subspace of holomorphic sections. It is usually referred as the "Bergman function". Note that away from the diagonal, the kernel $\sum_{i=1}^{N+1}\left\langle s_{i}\left(p_{1}\right), \cdot\right\rangle_{h^{k}} s_{i}\left(p_{2}\right)$ vanishes asymptotically, so the geometric information is carried only by $\rho_{k}(h)$.

In other words, the holomorphic embedding $\iota_{k}$ induced by the metric $\mathrm{Hilb}(h)$ gives a sequence of metrics by pull-back of the Fubini-Study metric $\iota_{k}^{*}\left(\omega_{\mathrm{FS}}\right)$, and this sequence converges to the initial metric $\omega$ as $k \rightarrow \infty$. We will also use the following technical result that can be proved with similar arguments to Theorem 2.1. See [Ze198, Cat99] and [Bou90] where the first term of the asymptotic expansion is identified.

Proposition 2.1. Let $(M, L)$ be a projective polarized manifold. Let $h \in \operatorname{Met}(L)$ be a metric whose curvature $c_{1}(h)=\omega>0$ is a Kähler form. Assume $\Omega>0$ is a volume form with continuous density. Then for $k \rightarrow \infty$ we have the asymptotic expansion

$$
\sum_{i=1}^{N+1}\left|s_{i}\right|_{h^{k}}^{2}=k^{n} \frac{\omega^{n}}{\Omega}+O\left(k^{n-1}\right)
$$

where $\left(s_{i}\right)$ is an orthonormal basis with respect to the $L^{2}$ inner product $\int_{M} h^{k}(\cdot, \cdot) \Omega=$ $\operatorname{Hilb}_{\Omega}\left(h^{k}\right)$. Here by $O\left(k^{n-1}\right)$ we mean that for $r \geq 0$,

$$
\left\|\sum_{i=1}^{N+1}\left|s_{i}\right|_{h^{k}}^{2}-k^{n} \frac{\omega^{n}}{\Omega}\right\|_{C^{r}} \leq c_{r} k^{n-1}
$$

where $c_{r}$ remains bounded if $h$ varies in a compact set (in smooth topology) in the space of hermitian metrics with positive curvature.

We will also need the following important technical result (see [LM07, Theorem 1], [Fin10, Theorem $7 \& 8$ ], [MM10, Section 6]).

Theorem 2.2. Consider $h \in \operatorname{Met}(L)$ with positive curvature and the operator on $C^{\infty}(M)$ given by

$$
Q_{k}(f)(p)=\frac{1}{k^{n}} \int_{M} \sum_{a, b}\left\langle s_{a}, s_{b}\right\rangle_{h^{k}}(q)\left\langle s_{a}, s_{b}\right\rangle_{h^{k}}(p) f(q) \Omega(q)
$$

which approximates the operator $\frac{\omega^{n}}{\Omega} \exp \left(-\frac{\Delta}{4 \pi k}\right)$ in the following sense. For any integer $r>0$, there exists $C>0$ such that for all $k \gg 0$ and any function $f \in C^{\infty}(X)$,

$$
\begin{aligned}
\left\|\left(\frac{\Delta}{k}\right)^{r}\left(Q_{k}(f)-\frac{\omega^{n}}{\Omega} \exp \left(-\frac{\Delta}{4 \pi k}\right) f\right)\right\|_{L^{2}} & \leq \frac{C}{k}\|f\|_{L^{2}}, \\
\| & Q_{k}(f)-\frac{\omega^{n}}{\Omega} f\left\|_{C^{r}} \leq \frac{C}{k}\right\| f \|_{C^{r+2}},
\end{aligned}
$$

where the norms are taken with respect to the induced Kähler form obtained from the fibrewise metric on the polarization $L$, and $\Delta$ is the Laplace operator for the induced Kähler metric. The estimate is uniform when the metric varies in a compact set of smooth hermitian metrics with positive curvature. 
We have the following first consequence.

Proposition 2.2. Let $h_{k} \in \operatorname{Met}\left(L^{k}\right)$ be a sequence of metrics such that $\omega_{k}:=$ $(1 / k) c_{1}\left(h_{k}\right)$ is convergent in smooth topology to the Kähler form $\omega$. Then the potentials $\beta_{k}=-\operatorname{tr}\left(\mu_{\Omega}^{0} \mu\right)$ (induced by the embeddings given by $\mathrm{Hilb}_{\Omega}\left(h_{k}\right)$ ) converge in smooth topology to the potential $1-\Omega / \omega^{n}$.

Note that given a Kähler form $\omega$, a sequence of Bergman metrics $h_{k}$ is known to exist by the previous theorem.

Proof. Let $\left(s_{i}\right)$ be an orthonormal basis of $H^{0}\left(L^{k}\right)$ with respect to the metric $H_{k}:=$ $\operatorname{Hilb}_{\Omega}\left(h_{k}\right)$. By the discussion at the beginning of Section 2, the balancing potential at $p \in M$ for the rescaled balancing flow is

$$
\beta_{k}\left(H_{k}\right)=-\int_{M} \sum_{a, b}\left(\frac{\left\langle s_{a}, s_{b}\right\rangle(q)}{\sum_{i=1}^{N+1}\left|s_{i}(q)\right|^{2}}-\frac{\delta_{a b}}{N+1}\right) \frac{\left\langle s_{a}, s_{b}\right\rangle(p)}{\sum_{i=1}^{N+1}\left|s_{i}(p)\right|^{2}} \Omega(q),
$$

where $\langle\cdot, \cdot\rangle$ stands for the fibrewise metric $h_{k}$. By the Riemann-Roch theorem, $N+1=$ $k^{n} \operatorname{Vol}_{L}(M)+O\left(k^{n-1}\right)$. From Proposition 2.1, the fact that $\omega_{k}$ is convergent to $\omega$ and the uniformity of the estimates, we obtain

$$
\begin{aligned}
\beta_{k}\left(H_{k}\right) & =1-\frac{k^{n}}{\sum_{i=1}^{N+1}\left|s_{i}(p)\right|^{2}} \int_{M} \sum_{a, b} \frac{\left\langle s_{a}, s_{b}\right\rangle(q)\left\langle s_{a}, s_{b}\right\rangle(p)}{k^{n}}\left(\frac{1}{\frac{\omega^{n}}{\Omega}(q)+O(1 / k)}\right) \Omega(q) \\
& =1-\frac{\Omega}{\omega^{n}} \int_{M} \frac{\left\langle s_{a}, s_{b}\right\rangle(q)\left\langle s_{a}, s_{b}\right\rangle(p)}{k^{n}}\left((1+O(1 / k)) \frac{\Omega}{\omega^{n}}(q)\right) \Omega(q)+O(1 / k) .
\end{aligned}
$$

But now, from Theorem 2.2, one knows the asymptotic behaviour of the quantification operator $Q_{k}(f)(p)=k^{-n} \int_{M} \sum_{a, b}\left\langle s_{a}, s_{b}\right\rangle(q)\left\langle s_{b}, s_{a}\right\rangle(p) f(q) \Omega(q)$.

Then, for $k \rightarrow \infty$, from (7) and the uniformity of the constants, one obtains

$$
\beta_{k}\left(H_{k}\right)(p)=1-\frac{\Omega}{\omega^{n}} Q_{k}\left(\frac{\Omega}{\omega^{n}}+O(1 / k)\right)+O(1 / k)
$$

The convergence of $Q_{k}\left(\Omega / \omega^{n}+O(1 / k)\right)$ to $1+O(1 / k)$ follows from the same arguments as in [Fin 10, pp. 10-11] and is a consequence of (6). This finally gives the desired result.

Independently of the flows considered, we also have a general result that complements Theorem 2.1.

Proposition 2.3. Let $h(t) \in \operatorname{Met}(L)$ be a path of hermitian metrics on $L$ with $c_{1}(h(t))>0$. Let $h_{k}(t)=\mathrm{FS}\left(\mathrm{Hilb}_{\Omega}\left(h(t)^{k}\right)\right)^{1 / k}$ be the path of induced Bergman metrics. Then $\partial h_{k}(t) / \partial t$ converges to $\partial h(t) / \partial t$ in $C^{\infty}$ topology as $k \rightarrow \infty$. This convergence is uniform if $h(t)$ belongs to a compact set in the space of positively curved hermitian metrics on $L$. 
Proof. The proof is essentially given in a discussion at the end of [Fin10, Section 1.4.1] for the sequence $\mathrm{FS}\left(\operatorname{Hilb}\left(h(t)^{k}\right)\right)^{1 / k}$. Assume that $h(t)=h_{0} e^{\phi_{t}}$ and that $\dot{\phi} e^{\phi_{t}} h_{0}$ is the infinitesimal change of the fibrewise metric, say at $t=0$. An infinitesimal change of the $L^{2}$ inner product corresponds to the hermitian matrix in the tangent space of the Bergman metrics

$$
A=\int_{M} k \dot{\phi}\left\langle s_{a}, s_{b}\right\rangle \Omega
$$

and thus the potential associated to that infinitesimal change is, after rescaling to $\operatorname{Met}(L)$,

$$
\frac{1}{k} \operatorname{tr}(A \mu)=\frac{1}{k} \int_{M} k \dot{\phi} \sum_{a, b}\left\langle s_{a}, s_{b}\right\rangle(p) \frac{\left\langle s_{a}, s_{b}\right\rangle(q)}{\sum_{i=1}^{N+1}\left|s_{i}(p)\right|^{2}} \Omega(q),
$$

where $\left(s_{i}\right)_{i=1}^{N+1}$ is an orthonormal basis of holomorphic sections with respect to $\operatorname{Hilb}_{\Omega}\left(h_{0}^{k}\right)$ and $h_{0}^{k}=\langle\cdot, \cdot\rangle$. Thus, using Proposition 2.1, one obtains

$$
\begin{aligned}
\frac{1}{k} \operatorname{tr}(A \mu)(p) & =\frac{\int_{M} \dot{\phi}(q) \sum_{a, b}\left\langle s_{a}, s_{b}\right\rangle(p)\left\langle s_{a}, s_{b}\right\rangle(q) \Omega(q)}{k^{n}\left(\frac{\omega_{0}^{n}}{\Omega}(p)+O(1 / k)\right)} \\
& =\frac{1}{\frac{\omega_{0}^{n}}{\Omega}(p)+O(1 / k)} Q_{k}(\dot{\phi})(p),
\end{aligned}
$$

and, as $k \rightarrow \infty$, this converges, by Theorem 2.2 , to $\dot{\phi}(p)$ after simplification.

Remark 2.2. Thus we have obtained the convergence of the family $h_{k}(t)$ in $C^{1}$ topology with respect to the variable $t$. Note that this result cannot be improved, in the sense that, by a direct computation, we do not expect convergence in $C^{2}$ topology. Let us be more precise. An infinitesimal change at order 2 of the induced $L^{2}$ inner product along a smooth path of the form $h_{0} e^{\phi_{t}}$ corresponds to a hermitian matrix

$$
B=\int_{M}\left((k \dot{\phi})^{2}+k \ddot{\phi}\right)\left\langle s_{a}, s_{b}\right\rangle \Omega .
$$

On the other hand, the potential associated to this infinitesimal change at $p \in M$ is given after rescaling by the formula

$$
\frac{1}{k}\left(\operatorname{tr}(B \mu)-\operatorname{tr}(A \mu)^{2}\right)(p) .
$$

Actually, if we write in an orthonormal basis the potential of the metric $\operatorname{FS}\left(\operatorname{Hilb}\left(h(t)^{k}\right)\right)$,

$$
\varphi(t)=\log \sum_{\alpha} \lambda_{\alpha}(t)\left|s_{\alpha}\right|^{2}
$$

with $\varphi(0)=\log \sum_{\alpha}\left|s_{\alpha}\right|^{2}$, then

$$
\ddot{\varphi}(t)_{\mid t=0}=\frac{\sum_{\alpha}\left(\lambda_{\alpha}\right)^{\prime \prime}(0)\left|s_{\alpha}\right|^{2}}{\sum_{\alpha}\left|s_{\alpha}\right|^{2}}-\left(\frac{\sum_{\alpha}\left(\lambda_{\alpha}\right)^{\prime}(0)\left|s_{\alpha}\right|^{2}}{\sum_{\alpha}\left|s_{\alpha}\right|^{2}}\right)^{2},
$$

which shows (8). In order to simplify the computations, assume that $h_{0}$ is a solution of the Calabi problem, i.e. $c_{1}\left(h_{0}\right)^{n}=\omega_{0}^{n}=\Omega$. Now, using this assumption, Proposition 2.1, 
and [MM07, Theorem 4.1.2], we obtain

$$
\frac{1}{k} \operatorname{tr}(B \mu)=\frac{1}{1+\frac{1}{4 \pi} \frac{\operatorname{scal}\left(\omega_{0}\right)}{2 k}+O\left(1 / k^{2}\right)} Q_{k}\left(k \dot{\phi}^{2}+\ddot{\phi}\right) .
$$

Then we can define an operator on $C^{\infty}(M)$ by

$$
\tilde{Q}_{k}(f)=\frac{1}{1+\frac{1}{4 \pi} \frac{\operatorname{scal}\left(\omega_{0}\right)}{2 k}} Q_{k}(f) .
$$

We write

$$
\frac{1}{k}\left(\operatorname{tr}(B \mu)-\operatorname{tr}(A \mu)^{2}\right)=\tilde{Q}_{k}(\ddot{\phi})+k\left(\tilde{Q}_{k}\left(\dot{\phi}^{2}\right)-\tilde{Q}_{k}(\dot{\phi})^{2}\right)+O(1 / k) .
$$

Then using Theorem 2.2 and [MM10, Theorem 6.1] which gives the asymptotic expansion of $Q_{k}$ at second order, $\frac{1}{k}\left(\operatorname{tr}(B \mu)-\operatorname{tr}(A \mu)^{2}\right)$ is equal to

$$
\begin{aligned}
\ddot{\phi}+O(1 / k)+\frac{1}{1+\frac{1}{4 \pi} \frac{\operatorname{scal}\left(\omega_{0}\right)}{2 k}} k\left(\dot{\phi}^{2}+\frac{1}{k}\left(\frac{\operatorname{scal}\left(\omega_{0}\right)}{8 \pi} \dot{\phi}^{2}-\frac{1}{4 \pi} \Delta_{\omega_{0}} \dot{\phi}^{2}\right)+O\left(1 / k^{2}\right)\right) \\
-\left(\frac{1}{1+\frac{1}{4 \pi} \frac{\operatorname{scal}\left(\omega_{0}\right)}{2 k}}\right)^{2} k\left(\dot{\phi}+\frac{1}{k}\left(\frac{\operatorname{scal}\left(\omega_{0}\right)}{8 \pi} \dot{\phi}-\frac{1}{4 \pi} \Delta_{\omega_{0}} \dot{\phi}\right)\right)^{2} \\
=\ddot{\phi}-\frac{1}{4 \pi} \Delta_{\omega_{0}} \dot{\phi}^{2}+2 \dot{\phi} \frac{1}{4 \pi} \Delta_{\omega_{0}} \dot{\phi}+O(1 / k)=\ddot{\phi}-\frac{1}{2 \pi}\|\nabla \dot{\phi}\|^{2},
\end{aligned}
$$

which is different from $\ddot{\phi}$.

We are now ready for the proof of Theorem 3 which identifies the limit of the sequence of rescaled $\Omega$-balancing flows for $k \rightarrow \infty$.

Proof of Theorem 3. We write $\omega_{t}=\omega+\sqrt{-1} \partial \bar{\partial} \phi_{t}$. By $C^{1}$ convergence in $t, \dot{\phi}_{t}$ is continuous and unique up to a constant that we shall fix by setting $\int_{M} \dot{\phi}_{t} \omega_{t}^{n}=0$. Consider the potential $\beta_{k}\left(\iota_{k}(t)\right)$ induced by the embedding $\iota_{k}(t)$ given by the rescaled $\Omega$-balancing flow at time $t$. Thanks to Proposition 2.3 and the fact that $\int_{M} \beta_{k}\left(\iota_{k}(t)\right) \omega_{k}^{n}(t) \rightarrow 0$ as $k \rightarrow \infty$, this sequence of potentials converges to $\dot{\phi}_{t}$. Moreover, using the balancing condition, we can apply Proposition 2.2 to get

$$
\dot{\phi}_{t}=\lim _{k \rightarrow \infty} \beta_{k}\left(\iota_{k}(t)\right)=1-\Omega / \omega_{t}^{n} .
$$

\section{The $\Omega$-Kähler flow and the proof of Theorem 2}

\subsection{The long time existence}

We are now interested in the flow

$$
\frac{\partial \phi_{t}}{\partial t}=1-\frac{\Omega}{\left(\omega+\sqrt{-1} \partial \bar{\partial} \phi_{t}\right)^{n}}
$$


over a compact Kähler manifold (not necessarily in an integral Kähler class), where $\phi_{0}=0$ and $\omega$ is a Kähler form in a fixed class $[\alpha]$. Of course, this can be rewritten as

$$
\left(\omega+\sqrt{-1} \partial \bar{\partial} \phi_{t}\right)^{n}=\frac{1}{1-\frac{\partial \phi_{t}}{\partial t}} e^{f} \omega^{n}
$$

where $f$ is a smooth (bounded) function defined by $f=\log \left(\Omega / \omega^{n}\right)$. We are interested in the long time existence of this flow and its convergence. We now study this, following the ideas of [Cao85]. Note that after we wrote this article we have been informed that similar results were proved recently in [FLM11], and we would like to thank Prof. Z. Błocki for pointing out this reference to us.

In this section we will prove the following result.

Theorem 3.1. Let $\phi_{t}$ be the solution of

$$
\frac{\partial \phi_{t}}{\partial t}=1-\frac{\Omega}{\left(\omega+\sqrt{-1} \partial \bar{\partial} \phi_{t}\right)^{n}}
$$

on the maximal time interval $0 \leq t<T_{\max }$. Let

$$
v_{t}=\phi_{t}-\frac{1}{\operatorname{Vol}_{L}(M)} \int_{M} \phi_{t} \omega^{n} .
$$

Then the $C^{\infty}$ norm of $v_{t}$ is uniformly bounded for all $0 \leq t<T_{\max }$, and $T_{\max }=+\infty$.

We remark that if we look at the formal level of this equation in terms of cohomology class, we obtain directly

which shows that the Kähler form

$$
\frac{\partial\left(\omega+\sqrt{-1} \partial \bar{\partial} \phi_{t}\right)}{\partial t}=0,
$$

$$
\omega_{t}:=\omega+\sqrt{-1} \partial \bar{\partial} \phi_{t}
$$

remains in the same class as $\omega+\sqrt{-1} \partial \bar{\partial} \phi_{0}$, i.e. $[\alpha]$.

Proposition 3.1. The functions $\frac{\partial \phi_{t}}{\partial t}$ and $\frac{1}{1-\partial \phi_{t} / \partial t}$ remain (uniformly) bounded in $C^{0}$ norm along the flow given by (10).

Proof. Differentiating (9), we obtain

$$
\frac{\partial}{\partial t}\left(\frac{\partial \phi_{t}}{\partial t}\right)=\frac{\Omega}{\omega_{t}^{n}} \Delta_{t}\left(\frac{\partial \phi_{t}}{\partial t}\right)
$$

with $\Delta_{t}$ the normalized Laplacian with respect to the metric $\omega+\sqrt{-1} \partial \bar{\partial} \phi_{t}$. We now apply the maximum principle for parabolic equations at the point where $\partial \phi_{t} / \partial t$ attains its maximum (respectively minimum). Plugging this information in (9), we obtain

$$
\frac{\partial \phi_{t}}{\partial t} \leq \sup _{M}\left(1-e^{f}\right)
$$

and moreover

$$
\frac{\partial \phi_{t}}{\partial t} \geq \inf _{M}\left(1-e^{f}\right)
$$


On the other hand,

$$
\frac{\partial}{\partial t}\left(\frac{1}{1-\frac{\partial \phi_{t}}{\partial t}}\right)=\frac{\left(\omega+\sqrt{-1} \partial \bar{\partial} \phi_{t}\right)^{n}}{\Omega} \Delta_{t}\left(\frac{\partial \phi_{t}}{\partial t}\right),
$$

and one applies again the maximum principle to obtain the proposition.

We denote by $\Delta$ the Laplacian with respect to the Kähler form $\omega$ given at $t=0$.

Lemma 3.1. One has $n+\Delta \phi_{t}>0$.

Proof. The fact that $\omega+\sqrt{-1} \partial \bar{\partial} \phi_{t}$ is a Kähler form implies by taking the trace that $n+\Delta \phi_{t}>0$.

We now show the upper bound for the Laplacian of the potential.

Proposition 3.2. There exist positive constants $C_{1}$ and $C_{2}$ such that

$$
0<n+\Delta \phi_{t} \leq C_{1} e^{C_{2}\left(\phi_{t}-\inf _{M \times[0, T)} \phi_{t}\right)} \quad \text { for all } t \in[0, T) .
$$

Proof. In the proof we denote $\phi_{t}$ by $\phi$, omitting the subscript for simplicity. Moreover, $g$ (resp. $g_{t}$ ) denotes the Riemannian metric associated to the Kähler form $\omega$ (resp. $\omega_{t}=$ $\left.\omega+\sqrt{-1} \partial \bar{\partial} \phi_{t}\right)$.

First of all, using holomorphic normal coordinates at any point $p \in M$, we have

$$
\Delta_{t}(n+\Delta \phi)=g_{t}^{k \bar{l}}\left(g^{i} \bar{j} \phi_{i \bar{j}}\right)_{k \bar{l}}=g_{t}^{k \bar{l}} R_{i \bar{j} k \bar{l}} \phi_{j \bar{i}}+g_{t}^{k \bar{l}} g^{i} \bar{j} \phi_{i \bar{j} k \bar{l}} \text {. }
$$

Set

$$
\hbar=\log \frac{\omega_{t}^{n}}{\Omega}=\log \omega_{t}^{n}-\log \omega^{n}-f
$$

so that $e^{-\hbar}=\Omega / \omega_{t}^{n}$. The idea of the proof is essentially to apply the maximum principle to the quantity $n+\Delta \phi$ with the operator $e^{-\hbar} \Delta_{t}-\partial / \partial t$.

Now, by using holomorphic normal coordinates and direct computations, we get

$$
\Delta \hbar=-g_{t}^{i \bar{q}} g_{t}^{p \bar{j}} \phi_{i \bar{j} k} \phi_{p \bar{q} \bar{k}}+g_{t}^{i \bar{j}}\left(-R_{i \bar{j}}+\phi_{i \bar{j} k \bar{k}}\right)+R-\Delta f .
$$

Here $R_{i} \bar{j} k \bar{l}$ and $R=\operatorname{scal}(\omega)$ denote the curvature tensor and the scalar curvature of the metric $g_{i \bar{j}}$ respectively. Then

$$
\begin{aligned}
\frac{\partial}{\partial t}(n+\Delta \phi) & =\Delta\left(\frac{\partial \phi}{\partial t}\right)=-\Delta\left(e^{-\hbar}\right)=e^{-\hbar}\left(\Delta \hbar-|\nabla \hbar|^{2}\right) \\
& =e^{-\hbar}\left(g_{t}^{i \bar{j}} g^{k \bar{l}} \phi_{i \bar{j} k \bar{l}}-g_{t}^{i \bar{j}} R_{i \bar{j}}+R-\Delta f-g_{t}^{i \bar{q}} g_{t}^{p \bar{j}} \phi_{i \bar{j} k} \phi_{p \bar{q} \bar{k}}-|\nabla \hbar|^{2}\right) .
\end{aligned}
$$

Thus

$$
\begin{aligned}
\left(e^{-\hbar} \Delta_{t}-\frac{\partial}{\partial t}\right)(n+\Delta \phi)= & e^{-\hbar}\left[g_{t}^{k \bar{l}} g^{i} \bar{j}\left(\phi_{i \bar{j} k \bar{l}}-\phi_{k \bar{l} i \bar{j}}\right)+g_{t}^{k \bar{l}} R_{i \bar{j} k \bar{l}} \phi_{j \bar{i}}\right. \\
& \left.+g_{t}^{i \bar{j}} R_{i \bar{j}}-R+\Delta f+g_{t}^{i \bar{q}} g_{t}^{p \bar{j}} \phi_{i \bar{j} k} \phi_{p \bar{q} \bar{k}}+|\nabla \hbar|^{2}\right] .
\end{aligned}
$$


On the other hand, since the covariant derivatives commute, we have

$$
\phi_{i \bar{j} k \bar{l}}-\phi_{k \bar{l} \bar{j}}=R_{i \bar{q} k \bar{l}} \phi_{q \bar{j}}-R_{i \bar{j} k \bar{q}} \phi_{q \bar{l}} .
$$

Hence

$$
\begin{aligned}
\left(e^{-\hbar} \Delta_{t}-\frac{\partial}{\partial t}\right)(n+\Delta \phi)= & e^{-\hbar}\left[2 g_{t}^{k k \bar{l}} R_{i \bar{j} k \bar{l}} \phi_{j \bar{i}}-g_{t}^{k \bar{l}} R_{k \bar{q}} \phi_{q \bar{l}}\right. \\
& \left.+g_{t}^{i \bar{j}} R_{i \bar{j}}-R+\Delta f+g_{t}^{i \bar{q}} g_{t}^{p \bar{j}} \phi_{i \bar{j} k} \phi_{p \bar{q} \bar{k}}+|\nabla \hbar|^{2}\right] .
\end{aligned}
$$

Moreover, if we choose another coordinate system so that $g_{i \bar{j}}=\delta_{i \bar{j}}$ and $\phi_{i \bar{j}}=\phi_{i \bar{i}} \delta_{i \bar{j}}$,

$$
\begin{aligned}
g_{t}^{k \bar{l}} R_{i \bar{j} k \bar{l}} \phi_{j \bar{i}}-g_{t}^{k \bar{l}} R_{k \bar{q}} \phi_{q \bar{l}} & =\sum_{i, k} R_{i \bar{i} k \bar{k}}\left(\frac{\phi_{i \bar{i}}}{1+\phi_{k \bar{k}}}-\frac{\phi_{k \bar{k}}}{1+\phi_{k \bar{k}}}\right) \\
& =\sum_{i, k} R_{i \bar{i} k \bar{k}} \frac{\phi_{i \bar{i}}^{2}-\phi_{i \bar{i}} \phi_{k \bar{k}}}{\left(1+\phi_{i \bar{i}}\right)\left(1+\phi_{k \bar{k}}\right)} \\
& =\frac{1}{2} \sum_{i, k} R_{i \bar{i} k \bar{k}} \frac{\left(\phi_{i \bar{i}}-\phi_{k \bar{k}}\right)^{2}}{\left(1+\phi_{i \bar{i}}\right)\left(1+\phi_{k \bar{k}}\right)},
\end{aligned}
$$

and

$$
\begin{aligned}
g_{t}^{k \bar{l}} R_{i \bar{j} k l} \phi_{j \bar{i}}+g_{t}^{i \bar{j}} R_{i \bar{j}}-R & =\sum_{i, k} R_{i \bar{i} k \bar{k}}\left(\frac{\phi_{i \bar{i}}}{1+\phi_{k \bar{k}}}+\frac{1}{1+\phi_{k \bar{k}}}-1\right) \\
& =\frac{1}{2} \sum_{i, k} R_{i \bar{i} k \bar{k}} \frac{\left(\phi_{i \bar{i}}-\phi_{k \bar{k}}\right)^{2}}{\left(1+\phi_{i \bar{i}}\right)\left(1+\phi_{k \bar{k}}\right)} .
\end{aligned}
$$

Therefore,

$$
\begin{aligned}
\left(e^{-\hbar} \Delta_{t}\right. & \left.-\frac{\partial}{\partial t}\right)(\Delta \phi) \\
& =e^{-\hbar}\left[\sum_{i, k} \frac{R_{i \bar{i} k \bar{k}}\left(\phi_{i \bar{i}}-\phi_{k \bar{k}}\right)^{2}}{\left(1+\phi_{i \bar{i}}\right)\left(1+\phi_{k \bar{k}}\right)}+\Delta f+g_{t}^{i \bar{q}} g_{t}^{p \bar{j}} \phi_{i \bar{j} k} \phi_{p \bar{q} \bar{k}}+|\nabla \hbar|^{2}\right] .
\end{aligned}
$$

Now, we assume the curvature tensor $R_{i \bar{j} k \bar{l}}$ is bounded below by $-C_{0}$, for some constant $C_{0}>0$, so that

$$
R_{i \bar{j} k \bar{l}} \geq-C_{0}\left(g_{i \bar{j}} g_{k \bar{l}}+g_{i \bar{l}} g_{k \bar{j}}\right) .
$$

Then, from (11) we obtain

$$
\left(e^{-\hbar} \Delta_{t}-\frac{\partial}{\partial t}\right)(\Delta \phi) \geq e^{-\hbar}\left[-2 C_{0}\left(\sum_{i, k} \frac{1+\phi_{i \bar{i}}}{1+\phi_{k \bar{k}}}-n^{2}\right)+\Delta f+g_{t}^{i \bar{q}} g_{t}^{p \bar{j}} \phi_{i \bar{j} k} \phi_{p \bar{q} \bar{k}}\right] .
$$


Finally, we consider the function $e^{-C \phi}(n+\Delta \phi)$ and compute

$$
\begin{aligned}
\Delta_{t}\left(e^{-C \phi}(n+\Delta \phi)\right)= & C^{2} e^{-C \phi}(n+\Delta \phi) g_{t}^{i \bar{j}} \phi_{i} \phi_{\bar{j}}-C e^{-C \phi} g_{t}^{i \bar{j}}\left[\phi_{i}(\Delta \phi)_{\bar{j}}+(\Delta \phi)_{i} \phi_{\bar{j}}\right] \\
& -C e^{-C \phi}(n+\Delta \phi) \Delta_{t} \phi+e^{-C \phi} \Delta_{t}(n+\Delta \phi) \\
\geq & -(n+\Delta \phi)^{-1} e^{-C \phi} g_{t}^{i \bar{j}}(\Delta \phi)_{i}(\Delta \phi)_{\bar{j}} \\
& -C e^{-C \phi}(n+\Delta \phi) \Delta_{t} \phi+e^{-C \phi} \Delta_{t}(n+\Delta \phi)
\end{aligned}
$$

and

$$
\frac{\partial}{\partial t}\left(e^{-C \phi}(n+\Delta \phi)\right)=-C e^{-C \phi}(n+\Delta \phi) \frac{\partial}{\partial t} \phi+e^{-C \phi} \frac{\partial}{\partial t}(n+\Delta \phi) .
$$

Thus,

$$
\begin{aligned}
\left(e^{-\hbar} \Delta_{t}-\frac{\partial}{\partial t}\right)\left(e^{-C \phi}(n+\Delta \phi)\right) \geq & -(n+\Delta \phi)^{-1} e^{-(C \phi+\hbar)} g_{t}^{i \bar{j}}(\Delta \phi)_{i}(\Delta \phi)_{\bar{j}} \\
& +e^{-C \phi}\left(e^{-\hbar} \Delta_{t}-\frac{\partial}{\partial t}\right)(n+\Delta \phi) \\
& -C e^{-C \phi}(n+\Delta \phi)\left(e^{-\hbar} \Delta_{t}-\frac{\partial}{\partial t}\right) \phi .
\end{aligned}
$$

Now observe that, by using $g_{i \bar{j}}=\delta_{i \bar{j}}, \phi_{i \bar{j}}=\phi_{i \bar{i}} \delta_{i \bar{j}}$ and (12), we have

$$
\begin{aligned}
&-(n+\Delta \phi)^{-1} g_{t}^{i \bar{j}}(\Delta \phi)_{i}(\Delta \phi)_{\bar{j}}+\left(\Delta_{t}-\frac{\partial}{\partial t}\right)(n+\Delta \phi) \\
& \geq-(n+\Delta \phi)^{-1} \sum_{i}\left(1+\phi_{i \bar{i}}\right)^{-1}\left|\sum_{k} \phi_{k \bar{k} i}\right|^{2} \\
& \quad+\sum_{i, j, k}\left(1+\phi_{i \bar{i}}\right)^{-1}\left(1+\phi_{k \bar{k}}\right)^{-1}\left|\phi_{i \bar{j} k}\right|^{2}+\Delta f-2 C_{0}\left(\sum_{i, k} \frac{1+\phi_{i \bar{i}}}{1+\phi_{k \bar{k}}}-n^{2}\right) \\
& \geq-2 C_{0}\left(\sum_{i, k} \frac{1+\phi_{i \bar{i}}}{1+\phi_{k \bar{k}}}-n^{2}\right)+\Delta f .
\end{aligned}
$$

Therefore, by taking $C=C_{0}+1$,

$$
\begin{aligned}
\left(e^{-\hbar} \Delta_{t}-\frac{\partial}{\partial t}\right)\left(e^{-C \phi}(n+\Delta \phi)\right) \geq e^{-(C \phi+\hbar)}\left(\Delta f+n^{2} C_{0}\right) \\
\quad-C e^{-(C \phi+\hbar)}(n+\Delta \phi)\left(n-e^{\hbar \frac{\partial \phi}{\partial t}}\right)+\left(C-C_{0}\right) e^{-(C \phi+\hbar)}(n+\Delta \phi) \sum_{i} \frac{1}{1+\phi_{i \bar{i}}} \\
\geq e^{-(C \phi+\hbar)}\left(\Delta f+n^{2} C_{0}\right)-C e^{-(C \phi+\hbar)}(n+\Delta \phi)\left(n-e^{\hbar} \frac{\partial \phi}{\partial t}\right) \\
\quad+e^{-(C \phi+\hbar+f /(n-1))}\left(1-\frac{\partial \phi}{\partial t}\right)^{-1 /(n-1)}(n+\Delta \phi)^{n /(n-1)},
\end{aligned}
$$


where in the last inequality we have used the arithmetic-geometric inequality

$$
\begin{aligned}
\sum_{i} \frac{1}{1+\phi_{i \bar{i}}} & \geq\left(\frac{\sum_{i}\left(1+\phi_{i \bar{i}}\right)}{\left(1+\phi_{1 \overline{1}}\right) \cdots\left(1+\phi_{n \bar{n}}\right)}\right)^{1 / n-1} \\
& =\left[e^{-f}\left(1-\frac{\partial \phi}{\partial t}\right)\right]^{1 /(n-1)}(n+\Delta \phi)^{1 /(n-1)} .
\end{aligned}
$$

Now the proposition follows from the maximum principle and Proposition 3.1. Actually, at the point $\left(p, t_{0}\right)$ where $e^{-C \phi}(n+\Delta \phi)$ achieves its maximum, the left hand side of (3.1) is nonpositive and hence

$$
\left(n+\Delta \phi\left(p, t_{0}\right)\right)^{n /(n-1)} \leq C^{\prime}\left(1+\left(n+\Delta \phi\left(p, t_{0}\right)\right)\right)
$$

with $C^{\prime}$ independent of $t$. Finally, $n+\Delta \phi\left(p, t_{0}\right) \leq C_{1}$, which gives the result.

Using the fact that we are working with plurisubharmonic potentials, we get the obvious fact:

Lemma 3.2. Denote

$$
v_{t}=\phi_{t}-\frac{1}{\operatorname{Vol}_{L}(M)} \int_{M} \phi_{t} \omega^{n}
$$

where $\phi_{t}$ is the solution to (10). Then there exist constants $c_{2}, c_{3}$ such that

$$
\sup _{M \times[0, T]} v_{t} \leq c_{2}, \quad \sup _{M \times[0, T]} \int_{M}\left|v_{t}\right| \omega^{n} \leq c_{3} .
$$

Proposition 3.3. There exists a constant $c_{4}>0$ such that

$$
\sup _{M \times[0, T]}\left|v_{t}\right| \leq c_{4} .
$$

Sketch of proof. We apply the Nash-Moser iteration argument. The only major difference with [Cao85, Lemma 3] is that in [Cao85, (1.14)], the right hand side is bounded by the term

$$
n ! \int_{M} \frac{\left(-v_{t}\right)^{p-1}}{p-1}\left(\frac{e^{f}}{1-\frac{\partial \phi_{t}}{\partial t}}-1\right) \omega^{n}
$$

But now, from Proposition 3.1, one can give the following upper bound for this term:

$$
C \int_{M} \frac{\left(-v_{t}\right)^{p-1}}{p-1} \omega^{n}
$$

where $C$ is a uniform positive constant. This ensures that one can apply the Nash-Moser argument to obtain the $C^{0}$ estimate in a similar way to the computations of [Cao85, p. 364].

With Propositions 3.3 and 3.2 and Lemma 3.1, one obtains a uniform bound of the quantity $n+\Delta \phi_{t}=n+\Delta v_{t}$. This implies, from the Schauder estimates, a first order estimate

$$
\sup _{M \times[0, T]}\left|\nabla v_{t}\right| \leq c_{5}\left(\sup _{M \times[0, T]}\left|\Delta v_{t}\right|+\sup _{M \times[0, T]}\left|v_{t}\right|\right) \leq c_{5}^{\prime} .
$$


All the second order derivatives of the potential $v_{t}$ are bounded. From the last inequality, one sees that in normal coordinates, the term $1+\phi_{i \bar{i}}$ is bounded from above, while from Proposition 3.3 and (3), the term $\prod_{i}\left(1+\phi_{i \bar{i}}\right)$ is bounded. So finally, $1+\phi_{i \bar{i}}$ is uniformly bounded along the time.

From Calabi's work and similarly to [Yau78, Cao85], it is now standard that this implies also the third order estimate. Finally, using Schauder's regularity theory [GT01] we have proved long time existence of the $\Omega$-Kähler flow. This concludes the proof of Theorem 3.1.

\subsection{The convergence}

In this section, we are interested in the convergence of the $\Omega$-Kähler flow.

Theorem 3.2. Denote $v_{t}=\phi_{t}-\left(1 / \operatorname{Vol}_{L}(M)\right) \int_{M} \phi_{t} \omega^{n}$ where $\phi_{t}$ is the solution to (10), the $\Omega$-Kähler flow. Then as $t \rightarrow \infty$, $v_{t}$ converges to $v_{\infty}$ in smooth topology and $\partial \phi_{t} / \partial t$ converges to a constant in smooth topology.

Note that [FLM11] gives an independent proof of this result. To prove the convergence of the $\Omega$-Kähler flow, we need some results of P. Li and S.-T. Yau on positive solutions of the heat equation on Riemannian compact manifolds [LY86, Section 2]. This takes the following form.

Proposition 3.4. Let $M$ be a compact manifold of dimension $n$. Let $\gamma_{i j}(t)$ be a family of Riemannian metrics on $M$ such that

- $c_{0} \gamma_{i j}(0) \leq \gamma_{i j}(t) \leq c_{0}^{\prime} \gamma_{i j}(0)$,

- $\left|\frac{\partial \gamma_{i j}}{\partial t}\right|(t) \leq c_{1} \gamma_{i j}(0)$,

- for the Ricci curvature, $R_{i j}(t) \geq-K g_{i j}(0)$,

where $c_{0}, c_{0}^{\prime}, c_{1}, K$ are positive constants independent of $t$. If we denote by $\tilde{\Delta}_{t}$ the Laplace operator of the metric $\gamma_{i j}(t)$, and if $\phi(p, t)$ is a positive solution of the equation

$$
\left(\tilde{\Delta}_{t}-\frac{\partial}{\partial t}\right) \phi(p, t)=0
$$

on $M \times[0, T)$, then one has the following Harnack type inequality for any $\alpha>1$ :

$$
\sup _{p \in M} \phi\left(p, t_{1}\right) \leq \inf _{p \in M} \phi\left(p, t_{2}\right)\left(\frac{t_{2}}{t_{1}}\right)^{n / 2} \exp \left(\frac{c_{3}}{t_{2}-t_{1}}+c_{4}\left(t_{2}-t_{1}\right)\right)
$$

where $c_{3}$ depends on $c_{0}^{\prime}$ and the diameter of $M$ with respect to $\gamma_{i j}(0)$, and $c_{4}$ depends on $\alpha, K, n, c_{0}^{\prime}, c_{1}, \sup \left\|\nabla^{2} \log \phi\right\|$ and $0<t_{1}<t_{2}<T$.

With Theorem 3.1 in hand, we shall apply Proposition 3.4 with $\gamma_{i j}(t)=\left(\omega_{t}^{n} / \Omega\right) g_{i \bar{j}}(t)$ where $g_{i} \bar{j}(t)$ is the metric associated with the Kähler form $\omega+\sqrt{-1} \partial \bar{\partial} \phi_{t}$. Thus, $\tilde{\Delta}_{t}=$ $\frac{\Omega}{\left(\omega+\sqrt{-1} \partial \bar{\partial} \phi_{t}\right)^{n}} \Delta_{t}$ and the potential $\phi_{t}$ solving (3) satisfies

$$
\left(\tilde{\Delta}_{t}-\frac{\partial}{\partial t}\right) \frac{\partial \phi_{t}(p)}{\partial t}=0
$$


The same reasoning as in [Cao85, Section 2] shows that the quantity

$$
E(t)=\int_{M}\left(\frac{\partial \phi_{t}}{\partial t}-\frac{1}{\operatorname{Vol}_{L}(M)} \int_{M} \frac{\partial \phi_{t}}{\partial t} \omega_{t}^{n}\right)^{2} \omega_{t}^{n}
$$

is decreasing to 0 (at least exponentially fast). The only difference with the computation in [Cao85] is that we need to show that the $\gamma_{i j}(t)$ are uniformly equivalent to $\gamma_{i j}(0)$. But this is clear because the metrics $g_{i}(t)$ and $g_{i}(0)$ are uniformly equivalent thanks to Theorem 3.1 , and the same holds for their respective volume forms. So the first eigenvalue of the Laplacian $\tilde{\Delta}_{t}$ is under control.

Similarly to [Cao85, Proposition 2.2], we now obtain Theorem 3.2. Note that a consequence of Theorem 3.1 is the existence of a sequence $v\left(p, t_{n}\right)$ (with $t_{n} \rightarrow \infty$ as $n \rightarrow \infty$ ), converging to a smooth function $v_{\infty}$ in smooth topology.

\subsection{Corollaries}

A direct consequence of Theorem 3.2 is the convergence of the $\Omega$-Kähler flow to the solution of the Calabi conjecture. Actually, the limit $v_{\infty}$ satisfies

$$
\left(\omega+\sqrt{-1} \partial \bar{\partial} v_{\infty}\right)^{n}=\left(\omega+\sqrt{-1} \partial \bar{\partial} \phi_{\infty}\right)^{n}=\Omega .
$$

In other words, one can prescribe the volume form in a given Kähler class. This was first proved by S.-T. Yau in [Yau78] and our proof uses essentially the same type of estimates. Of course, if $M$ has first Chern class trivial, then the limit metric is a Calabi-Yau metric.

We also remark that one can modify (9) slightly if the manifold $M$ has first Chern class negative. In that case, it is natural to introduce the following flow:

$$
\left(\omega+\sqrt{-1} \partial \bar{\partial} \phi_{t}\right)^{n}=\frac{1}{1-\frac{\partial \phi_{t}}{\partial t}} e^{f+\phi_{t}} \omega^{n}
$$

where $\omega \in-2 \pi c_{1}(M)>0$, and $f$ is the deviation of the Ricci curvature of $\omega$, that is,

$$
\operatorname{Ric}(\omega)+\omega=\sqrt{-1} \partial \bar{\partial} f \quad \text { and } \quad \int_{M} \frac{1}{1-\frac{\partial \phi_{t}}{\partial t}} e^{f+\phi_{t}} \omega^{n}=\operatorname{Vol}_{K_{M}}(M) .
$$

In that case computations similar to Section 3.1 will involve the operator $\Delta_{t}-$ Id since by differentiating (14), one obtains

$$
\frac{\partial}{\partial t}\left(\frac{\partial \phi_{t}}{\partial t}\right)=\frac{e^{f+\phi_{t}} \omega^{n}}{\omega_{t}^{n}}\left(\Delta_{t}\left(\frac{\partial \phi_{t}}{\partial t}\right)-\frac{\partial \phi_{t}}{\partial t}\right) .
$$

The uniform bound of the term $\frac{1}{1-\partial \phi_{t} / \partial t}$ can be proved in a similar way to Section 3.1 (Proposition 3.1), and by the maximum principle, there is a uniform bound of the potentials $\phi_{t}$. Thus, one obtains the convergence of $\phi_{t}$ as $t \rightarrow \infty$, and $\omega+\sqrt{-1} \partial \bar{\partial} \phi_{\infty}$ is a smooth Kähler-Einstein metric with negative curvature. 


\section{Proof of Theorem 1}

In this section, $(M, L)$ is a polarized manifold and we are only considering integral Kähler classes. The techniques we use to prove Theorem 1 are inspired by the techniques of [Fin10].

\subsection{First order approximation}

From the results of Section 3 we know that from any starting point $\omega=\omega_{0}$, there exists a solution

$$
\omega_{t}=\omega+\sqrt{-1} \partial \bar{\partial} \phi_{t}
$$

to the $\Omega$-Kähler flow. We can write $\omega_{t}=c_{1}\left(h_{t}\right)$ where $h_{t}$ is a sequence of hermitian metrics on the line bundle $L$. Furthermore, we can construct a natural sequence of Bergman metrics

$$
\hat{h}_{k}(t)=\mathrm{FS}\left(\operatorname{Hilb}_{\Omega}\left(h_{t}^{k}\right)\right)^{1 / k}
$$

by pulling back the Fubini-Study metric using sections which are orthonormal with respect to the inner product

$$
\frac{1}{k^{n}} \int_{M} h_{t}(\cdot, \cdot)^{k} \Omega
$$

Using Proposition 2.1 we obtain the asymptotic behaviour

$$
\hat{h}_{k}(t)=\left(\frac{k^{n} c_{1}\left(h_{t}\right)^{n}}{\Omega}+O(1 / k)\right)^{1 / k} h_{t}
$$

for $k \gg 1$. Thus, the sequence $\hat{h}_{k}(t)$ converges to $h_{t}$ as $k \rightarrow \infty$.

On the other hand, the rescaled $\Omega$-balancing flow provides a sequence of metrics $\omega_{k}(t)=c_{1}\left(h_{k}(t)\right)$ which are solutions to (2). Note that by construction, we fix $h_{k}(0)=$ $\hat{h}_{k}(0)$ for the starting point of the rescaled $\Omega$-balancing flow.

In this section, we wish to evaluate the distance between the two metrics $h_{k}(t)$ and $\hat{h}_{k}(t)$. Since we are dealing with algebraic metrics, we have the (rescaled) metric on hermitian matrices given by

$$
d_{k}\left(H_{0}, H_{1}\right)=\left(\frac{\operatorname{tr}\left(H_{0}-H_{1}\right)^{2}}{k^{2}}\right)^{1 / 2}
$$

on $\operatorname{Met}\left(H^{0}\left(L^{k}\right)\right)$ which induces a metric on $\operatorname{Met}(L)$, denoted by $\operatorname{dist}_{k}$.

Proposition 4.1. One has

$$
\operatorname{dist}_{k}\left(h_{k}(t), \hat{h}_{k}(t)\right) \leq C / k
$$

for some constant $C>0$ independent of $k$. 
Proof. The proof is similar to [Fin10, Proposition 10]. Let $e^{\phi(t)} h_{0}$ be a family of hermitian metrics with positive curvature, and denote

$$
\omega_{t}=c_{1}\left(e^{\phi(t)} h_{0}\right) .
$$

The infinitesimal change at $t$ in the $L^{2}$ inner product induced by this path and the volume form $\Omega$ is given by

$$
\hat{U}_{\alpha, \beta}(t)=\frac{1}{k^{n}} \int_{M}\left\langle s_{\alpha}, s_{\beta}\right\rangle k \dot{\phi}(t) \Omega
$$

for $\left(s_{\alpha}\right)$ an orthonormal basis of $H^{0}\left(L^{k}\right)$ with respect to the $L^{2}$ inner product

$$
\frac{1}{k^{n}} \int_{M} e^{k \phi(t)} \Omega
$$

The formula is obtained by noticing that the variation occurs with respect to the fibrewise metric. Now, if furthermore $\phi(t)$ is a solution to the $\Omega$-Kähler flow, this infinitesimal change is given at $\hat{h}_{k}(t)$ as

$$
\hat{U}_{\alpha, \beta}(t)=\frac{1}{k^{n}} \int_{M}\left\langle s_{\alpha}, s_{\beta}\right\rangle\left(k\left(1-\frac{\Omega}{\omega_{t}^{n}}\right)\right) \Omega,
$$

with $\left(s_{\alpha}\right)$ satisfying the same assumption as above.

On the other hand, the tangent (at the same point $\hat{h}_{k}(t)$ ) to the rescaled $\Omega$-balancing flow (2) is given directly by the moment map $\mu_{\Omega}^{0}$, and we write the infinitesimal change of the $L^{2}$ metric as

$$
U_{\alpha, \beta}(t)=k \int_{M}\left(\frac{\delta_{\alpha \beta}}{N+1}-\frac{\left\langle s_{\alpha}, s_{\beta}\right\rangle}{\sum_{\alpha=1}^{N+1}\left|s_{\alpha}\right|^{2}}\right) \Omega .
$$

Again, from Proposition 2.1, one has asymptotically

$$
U_{\alpha, \beta}(t)=\hat{U}_{\alpha, \beta}(t)+\frac{1}{k^{n}} \int_{M}\left\langle s_{\alpha}, s_{\beta}\right\rangle O(1) \Omega .
$$

Here $O(1)$ stands implicitly for a (smooth) function which is bounded independently of the variables $t$ and $k$. Thus, one has

$$
\frac{\operatorname{tr}\left(\hat{U}_{\alpha, \beta}(t)-U_{\alpha, \beta}(t)\right)^{2}}{k^{2}}=\left\langle\frac{1}{k} O(1), Q_{k}\left(\frac{1}{k} O(1)\right)\right\rangle_{L^{2}} .
$$

We can use inequality (6) of Theorem 2.2 to obtain

$$
\frac{\operatorname{tr}\left(\hat{U}_{\alpha, \beta}(t)-U_{\alpha, \beta}(t)\right)^{2}}{k^{2}}=O\left(k^{-2}\right) .
$$

This shows that $\left.d_{k}\left(\hat{U}_{\alpha, \beta}(t), U_{\alpha, \beta}(t)\right)\right)=O(1 / k)$. If we denote by $\tilde{h}_{k}(t)$ the rescaled balancing flow passing through $\hat{h}_{k}\left(t_{0}\right)$ at $t=t_{0}$, we have just proved that $\tilde{h}_{k}(t)$ and $\hat{h}_{k}(t)$ are tangent up to an $O(1 / k)$ error term at $t=t_{0}$. On the other hand, it is clear that $\tilde{h}_{k}(t)$ 
and $h_{k}(t)$ are close when $t \rightarrow \infty$, because they are obtained through the gradient flow of the same moment map and this gradient flow converges to the unique $\Omega$-balanced metric (this is a consequence of [Don09]). Thus $\operatorname{dist}\left(\tilde{h}_{k}(t), h_{k}(t)\right)=O(1 / k)$. This finally proves the result.

\subsection{Higher order approximations}

In this section, we improve the result of the last section by constructing a new timedependent function

$$
\psi(k, t)=\phi_{t}+\sum_{j=1}^{m} \frac{1}{k^{j}} \eta_{j}(t)
$$

which is obtained by deforming the solution to the $\Omega$-Kähler flow and which has the property of being "as close as we wish" to the $\Omega$-balancing flow. We will need to compare this metric to the Bergman metric $h_{k}(t)$. Thus, we introduce the Bergman metric associated to $h_{0} e^{\psi(k, t)}$, i.e.

$$
\bar{h}_{k}(t)=\mathrm{FS}\left(\operatorname{Hilb}_{\Omega}\left(h_{0}^{k} e^{k \psi(k, t)}\right)\right)^{1 / k} .
$$

We wish to minimize the quantity $\operatorname{dist}_{k}\left(\bar{h}_{k}(t), h_{k}(t)\right)$ by showing an estimate of the form $\operatorname{dist}_{k}\left(\bar{h}_{k}(t), h_{k}(t)\right)<C / k^{m+1}$, with $C>0$ a constant independent of $k \gg 0$ and $t$. This is the parameter version of [Don01, Theorem 26], and Proposition 4.1 shows that the result holds for $m=0$. One needs to choose inductively the functions $\eta_{j}$, and this is done by linearizing the Monge-Ampère operator.

Let us give some details for the first step of the induction, that is, to find $\eta_{1}$. Similarly to (15), the tangent to the path $\bar{h}_{k}(t)$ can be written as

$$
\bar{T}_{\alpha \beta}(t)=\frac{1}{k^{n}} \int_{M} k\left\langle s_{\alpha}, s_{\beta}\right\rangle\left(1-\frac{\Omega}{\omega_{t}^{n}}+\frac{\dot{\eta_{1}}}{k}+O(1 / k)\right) \Omega,
$$

where $\omega_{t}=\omega+\sqrt{-1} \partial \bar{\partial} \phi_{t}$ and $\left(s_{\alpha}\right)$ is $L^{2}$ orthonormal with respect to $e^{\phi_{t}} h_{0}$ and the volume form $\Omega$. On the other hand, the tangent to the rescaled balancing flow at the point $\bar{h}_{k}(t)$ is given, similarly to $(16)$, by

$$
T_{\alpha \beta}(t)=\frac{1}{k^{n}} \int_{M} k\left\langle s_{\alpha}, s_{\beta}\right\rangle\left(1-\frac{\Omega}{c_{1}\left(\bar{h}_{k}(t)\right)^{n}}+O(1 / k)\right) \Omega .
$$

But now,

$$
\frac{\Omega}{c_{1}\left(\bar{h}_{k}(t)\right)^{n}}=\frac{\Omega}{\omega_{t}^{n}}-\frac{\Omega}{\omega_{t}^{n}} \Delta_{t}\left(\frac{1}{k} \eta_{1}\right)+O\left(1 / k^{2}\right),
$$

and we can write the error term $k O(1 / k)$ from (17) as

$$
k O(1 / k)=\sum_{i \geq 0} \gamma_{1, i} k^{-i}=\gamma_{1,0}+O(1 / k)
$$


with $\gamma_{1, i}$ smooth real-valued functions depending on the metric and obtained from the Bergman function asymptotics, so

$$
T_{\alpha \beta}(t)=\frac{1}{k^{n}} \int_{M}\left\langle s_{\alpha}, s_{\beta}\right\rangle\left(k\left(1-\frac{\Omega}{\omega_{t}^{n}}+\frac{\Omega}{\omega_{t}^{n}} \Delta_{t}\left(\frac{1}{k} \eta_{1}\right)+\frac{\gamma_{1,0}}{k}\right)+O(1 / k)\right) \Omega .
$$

If we wish to force $d_{k}\left(\bar{T}_{\alpha \beta}(t), T_{\alpha \beta}(t)\right)$ to be of size $O\left(1 / k^{2}\right)$, we need to find $\eta_{1}$ such that

$$
\frac{\partial \eta_{1}(t)}{\partial t}-\frac{\Omega}{\omega_{t}^{n}} \Delta_{t} \eta_{1}(t)=\gamma_{1,0}
$$

for all $t \geq 0$ and $\eta_{1}(0)=0$. But, by standard parabolic theory (see, e.g., [Bak11, Section 3.1] for a detailed exposition), a smooth solution $\eta_{1}$ to the above initial-value problem exists and is unique. Hence

$$
\frac{\operatorname{tr}\left(\bar{T}_{\alpha \beta}(t)-T_{\alpha \beta}(t)\right)^{2}}{k^{2}}=\left\langle O\left(1 / k^{2}\right), Q_{k}\left(O\left(1 / k^{2}\right)\right)\right\rangle_{L^{2}}
$$

and we can conclude with similar arguments to Section 4.1: there exists a constant $C>0$ independent of $t$ such that

$$
\frac{\operatorname{tr}\left(\bar{T}_{\alpha \beta}(t)-T_{\alpha \beta}(t)\right)^{2}}{k^{2}} \leq \frac{C}{k^{4}} .
$$

This implies, by the same arguments as at the end of the proof of Proposition 4.1, that

$$
\operatorname{dist}_{k}\left(h_{k}(t), \bar{h}_{k}(t)\right) \leq C / k^{2} .
$$

Now, for higher order expansions, one considers higher order asymptotics in the expressions above. The same reasoning can be applied to construct higher order approximation. Knowing the terms $\eta_{1}, \ldots, \eta_{m}$ one has to find $\eta_{m+1}$ solving a similar equation to (18), where the (nonconstant) RHS will depend on the functions $\eta_{j}(1 \leq j \leq m)$ computed at the previous step:

$$
\frac{\partial \eta_{m+1}(t)}{\partial t}-\frac{\Omega}{\omega_{t}^{n}} \Delta_{t} \eta_{m+1}(t)=\gamma_{m+1,0}\left(\eta_{1}, \ldots, \eta_{m}\right) .
$$

Again, it is possible to solve (20) by inverting the operator $\frac{\Omega}{\omega_{t}^{n}} \Delta_{t}-\frac{\partial}{\partial t}$. Finally, we have obtained

Theorem 4. Given the solution $\phi_{t}$ to the $\Omega$-Kähler flow (3) and $k \gg 0$, there exist functions $\eta_{1}, \ldots, \eta_{m}, m \geq 1$, such that the deformation of $\phi_{t}$ given by the potential

$$
\psi(k, t)=\phi_{t}+\sum_{j=1}^{m} \frac{1}{k^{j}} \eta_{j}(t)
$$

satisfies

$$
\operatorname{dist}_{k}\left(h_{k}(t), \bar{h}_{k}(t)\right) \leq C / k^{m+1} .
$$

Here $\bar{h}_{k}(t)=\operatorname{FS}\left(\operatorname{Hilb}_{\Omega}\left(h_{0}^{k} e^{k \psi(k, t)}\right)\right)^{1 / k} \in \operatorname{Met}(L)$ is the induced Bergman metric from the potential $\psi, h_{k}(t) \in \operatorname{Met}(L)$ is the sequence of metrics obtained by the rescaled balancing flow (2), and $C$ is a positive constant independent of $k$ and $t$. 
Proof. The only point that we did not explain earlier is that $C$ is independent of $t \in \mathbb{R}_{+}$. This comes from the following facts. On one hand, the expansion of the Bergman function of a family of metrics $h_{t}$ is uniform if the metrics $h_{t}$ belong to a compact subset of hermitian positive metrics in $\operatorname{Met}(L)$ (see Theorem 2.1). On the other hand, we have seen that the metrics involved in the $\Omega$-Kähler flow are in a bounded set, since $\omega_{t}$ is convergent in smooth topology as $t \rightarrow \infty$ thanks to Theorem 3.2. This completes the proof of Theorem 4.

Furthermore, one can slightly improve this result by showing that one has $C^{1}$ convergence in $t$.

Proposition 4.2. Under the same assumptions and notations of the previous theorem, one has

$$
\operatorname{dist}_{k}\left(\frac{\partial h_{k}(t)}{\partial t}, \frac{\partial \bar{h}_{k}(t)}{\partial t}\right) \leq \frac{C}{k^{m}}
$$

where $C$ is a constant uniform in $k$ and $t$.

Proof. One needs essentially to give an estimate of the quantity

$$
\operatorname{tr}\left(\frac{\partial \bar{T}_{\alpha \beta}(t)}{\partial t}-\frac{\partial T_{\alpha \beta}(t)}{\partial t}\right)^{2}
$$

Assume that we have fixed $\eta_{1}$ as in the proof of Theorem 4, that is, $m=1$. Then, as the first step, we are led to estimate

$$
\begin{aligned}
& \frac{1}{k^{n}} \int_{M}\left\langle s_{\alpha}, s_{\beta}\right\rangle k\left(\frac{\partial}{\partial t}\left(\frac{\Omega}{\omega_{t}^{n}} \Delta_{t}\left(\eta_{1} / k\right)-\dot{\eta}_{1} / k+\gamma_{1,0} / k+O\left(1 / k^{2}\right)\right)\right) \Omega \\
& \quad+\frac{1}{k^{n}} \int_{M} k\left(k \frac{\partial \phi_{t}}{\partial t}\right)\left\langle s_{\alpha}, s_{\beta}\right\rangle\left(\frac{\Omega}{\omega_{t}^{n}} \Delta_{t}\left(\eta_{1} / k\right)-\dot{\eta}_{1} / k+\gamma_{1,0} / k+O\left(1 / k^{2}\right)\right) \Omega .
\end{aligned}
$$

In (21), the term $O\left(1 / k^{2}\right)$ stands for a smooth function $r(p, k, t)$, where $p \in M$, uniformly bounded over $M$ and in the variable $t$. But we know that the asymptotics of the Bergman kernel is given by polynomial expressions in the curvature and its covariant derivative. We can write $r(p, k, t)=\sum_{i \geq 2} k^{-i} r_{i}(p, t)$ where $r_{i}(p, t)$ are smooth in $t$ and $p$. Thus $\|r(p, k, t)\|_{C^{\infty}(M)}<C_{1} / k^{2}$ and $\|\partial r(p, k, t) / \partial t\|_{C^{\infty}(M)}<C_{2} / k^{2}$, where $C_{1}, C_{2}$ do not depend on $k, t$ or $p$. The independence from $t$ is again obtained from the fact that the metric $\omega_{t}$ along the $\Omega$-Kähler flow is convergent (Theorem 3.2) and from the uniformity of the expansion. Moreover, since $\eta_{1}$ is a smooth solution of (18) in $t$, the term (21) is uniformly bounded by $C_{3} / k^{2}$ by the same argument as in the proof of Theorem 4 (inequality (19)) . On other hand, by the same reasoning, (22) is uniformly bounded by $C_{4} / k$, where $C_{4}$ is independent of $t$ and $k$. This provides the result for $m=1$.

The computations for $m>1$ are completely similar. Also, higher order derivatives in $t$ could be treated in a similar way. 


\section{3. $L^{2}$ estimates in finite-dimensional set-up}

We start this section by fixing some notations and giving some definitions. Let us fix a reference metric $\omega_{0} \in 2 \pi c_{1}(L)$. We denote by $\tilde{\omega}_{0}=k \omega_{0}$ the induced metric in $2 \pi k c_{1}(L)$. We need the notion of $R$-bounded geometry in $C^{r}$ [Don01, Section 3.2]. We say that another metric $\tilde{\omega} \in 2 \pi k c_{1}(L)$ has $R$-bounded geometry in $C^{r}$ if $\tilde{\omega}>(1 / R) \tilde{\omega}_{0}$ and $\left\|\tilde{\omega}-\tilde{\omega}_{0}\right\|_{C^{r}\left(\tilde{\omega}_{0}\right)}<R$. We say that a basis $\left(s_{i}\right)$ of $H^{0}\left(M, L^{k}\right)$ is $R$-bounded if the FubiniStudy metric induced by the embedding of $M$ in $\mathbb{P} H^{0}\left(L^{k}\right)^{\vee}$ associated to the $\left(s_{i}\right)$ has $R$-bounded geometry.

The reason for working with $R$-bounded metrics is to avoid constants depending on $k$ in the forthcoming estimates. Let us fix

$$
H_{A}=\sum_{i, j} A_{i j}\left(s_{i}, s_{j}\right)=\operatorname{tr}(A \mu) \in C^{\infty}(M),
$$

where $A=\left(A_{i j}\right)$ is a hermitian matrix, $\left(s_{i}\right)$ is a basis of $H^{0}\left(L^{k}\right)$, and $(\cdot, \cdot)$ denotes the fibrewise Fubini-Study inner product induced by the basis $\left(s_{i}\right)$. This function corresponds to the potential obtained by an $A$-deformation of the Fubini-Study metric, i.e. when one is moving the Fubini-Study metric in a $\operatorname{Lie}(\mathrm{SU}(N+1))$ orbit. Moreover, we denote by $\|A\|_{\text {op }}=\max |A \zeta| /|\zeta|$ the operator norm, given by the maximum modulus of the eigenvalues of the hermitian matrix $A$, and by $\|A\|^{2}=\operatorname{tr}\left(A^{2}\right)=\operatorname{tr}\left(A A^{*}\right) \geq 0$ the HilbertSchmidt norm. We will need the following very general result:

Proposition 4.3 ([Don01, Lemma 24], [Fin10, Proposition 12]). There exists $C>0$, independent of $k$, such that for any basis $\left(s_{i}\right)$ of $H^{0}\left(L^{k}\right)$ with $R$-bounded geometry in $C^{r}$ and any hermitian matrix $A$,

$$
\left\|H_{A}\right\|_{C^{r}} \leq C\left\|\mu_{\Omega}(\iota)\right\|_{\text {op }}\|A\|
$$

where $\iota$ is the embedding induced by $\left(s_{i}\right)$.

Proof. By definition, $\mu_{\Omega}(\iota)=\int_{M} \mu(\iota) \Omega$. Given a holomorphic section $s$ of $L \rightarrow M$, one defines a holomorphic section $\tilde{s}$ of $\bar{L}^{*} \rightarrow \bar{M}$ (here $\bar{M}$ is just $M$ with the opposite complex structure) thanks to the bundle isomorphism given by the fibre metric. Then, for a hermitian matrix $A$, one can define the section $\sigma_{A}=\sum A_{i j} s_{i} \otimes \tilde{s}_{j}$ and compute its $L^{2}$ norm over $M \times \bar{M}$. This $L^{2}$ norm is given by $\operatorname{tr}\left(A \mu_{\Omega} \mu_{\Omega}^{*} A^{*}\right)^{1 / 2}$. But one has an obvious upper bound for that term, by a standard inequality: for hermitian matrices $G, F$, $\operatorname{tr}(F G F) \leq\|F\|^{2}\|G\|_{\text {op }}$. Thus,

$$
\left\|\sigma_{A}\right\|=\operatorname{tr}\left(A \mu_{\Omega} \mu_{\Omega}^{*} A^{*}\right)^{1 / 2} \leq\left\|\mu_{\Omega}(\iota)\right\|_{\text {op }}\|A\| .
$$

On the other hand, for any holomorphic section $\sigma$ of a hermitian vector bundle $\tilde{L} \rightarrow Y$, one has the $L^{2}$ estimate $\|\sigma\|_{C^{r}\left(Y^{\prime}\right)} \leq C\|\sigma\|_{L^{2}(Y)}$ for a submanifold $Y^{\prime} \subset Y$ and some constant $C$ that depends on $Y$. This is described in [Don01, Lemma 24]. Hence, applying this result with $Y=M \times \bar{M}$ and $Y^{\prime}=M$, together with (23), one obtains the desired inequality.

We will need the following lemma. 
Lemma 4.1. Fix $r \geq 2$. Assume that for all $t \in[0, T]$, the family of bases $\left\{s_{i}\right\}(t)$ of $H^{0}\left(L^{k}\right)$ have R-bounded geometry. Define by $h(t)$ the family of Bergman metrics induced by $\left\{s_{i}\right\}(t)$. Then the induced family of Fubini-Study metrics $\tilde{\omega}(t)$ satisfy

$$
\|\tilde{\omega}(0)-\tilde{\omega}(T)\|_{C^{r-2}}<C \sup _{t}\left\|\mu_{\Omega}(\iota(t))\right\|_{\text {op }} \int_{0}^{T} \operatorname{dist}(h(s), h(0)) d s,
$$

and also

$$
\begin{aligned}
\left\|\frac{\partial \tilde{\omega}}{\partial t}(0)-\frac{\partial \tilde{\omega}}{\partial t}(T)\right\|_{C^{r-2}}< & C^{*} \sup _{t}\left\|\mu_{\Omega}(\iota(t))\right\|_{\mathrm{op}} \int_{0}^{T} \operatorname{dist}\left(\frac{\partial h}{\partial s}(s), \frac{\partial h}{\partial s}(0)\right) d s \\
& +C^{*} \sup _{t}\left\|d \mu_{\Omega}(\iota(t))\right\|_{\mathrm{op}} \int_{0}^{T} \operatorname{dist}(h(s), h(0)) d s,
\end{aligned}
$$

where $C, C^{*}$ are constants uniform in $k$.

Proof. Thanks to [Fin10, Lemma 13], we just need to check the second inequality. For the deformation $A(t)$ of the $L^{2}$ metric induced along the path from 0 to $T$, one has

$$
\begin{aligned}
\left\|\frac{\partial^{2} \tilde{\omega}(t)}{\partial t^{2}}\right\|_{C^{r-2}} & =\left\|\sqrt{-1} \partial \bar{\partial} \frac{\partial}{\partial t} H_{A(t)}\right\|_{C^{r-2}} \\
& \leq\|\partial \bar{\partial} \operatorname{tr}(\dot{A}(t) \mu)\|_{C^{r-2}}+\left\|\partial \bar{\partial} \operatorname{tr}\left(A(t) \dot{\mu}\left(l_{t}\right)\right)\right\|_{C^{r-2}} .
\end{aligned}
$$

The first term of (25) can be bounded from above by $C\left\|\mu_{\Omega}(\iota(t))\right\|_{\text {op }}\|\dot{A}(t)\|$ using directly Proposition 4.3. For the second term, one needs to adapt the proof of Proposition 4.3, but this can be done with no major difficulty. Hence, the second term of (25) can be bounded from above by

$$
\begin{aligned}
\left\|\partial \bar{\partial} \operatorname{tr}\left(A(t) \dot{\mu}\left(\iota_{t}\right)\right)\right\|_{C^{r-2}} & \leq C\left\|\int_{M} \dot{\mu}(\iota(t)) \Omega\right\|_{\mathrm{op}}\|A(t)\| \\
& \leq C^{\prime}\left\|d \mu_{\Omega}(\iota(t))\right\|_{\mathrm{op}}\|A(t)\| .
\end{aligned}
$$

Then by integration, one obtains the desired estimate.

Corollary 4.1. Let $\tilde{\omega}_{k}$ be a sequence of metrics with $R / 2$-bounded geometry in $C^{r+2}$ such that the norms $\left\|\mu_{\Omega}\left(\tilde{\omega}_{k}\right)\right\|_{\text {op }}$ are uniformly bounded. Then there is a constant $C>0$ independent of $k$ such that if $\tilde{\omega}$ has $\operatorname{dist}_{k}\left(\tilde{\omega}, \tilde{\omega}_{k}\right)<C$, then $\tilde{\omega}$ has $R$-bounded geometry in $C^{r}$.

Proof. The proof is completely similar to [Fin10, Lemma 14].

\subsection{Projective estimates}

In this subsection, we aim to control the operator norm of the moment map in terms of the Riemannian distance in the Bergman space

$$
\mathcal{B}=\mathrm{GL}(N+1) / \mathrm{U}(N+1) .
$$


With this result in hand, we can consider the gradient flow of the moment map and show its convergence.

We start our investigation with the following result, which is a direct consequence of Theorem 2.1.

Proposition 4.4. Let $h$ be a hermitian metric on $L$ with curvature $\omega=c_{1}(h)>0$. Consider the sequence $h_{k}=\mathrm{FS}(\mathrm{Hilb}(h)) \in \operatorname{Met}\left(L^{k}\right)$ of Bergman metrics, approximating $h$ after renormalization, thanks to Theorem 2.1. Set

$$
I_{\Omega, k}=\int_{M}\left\langle s_{i}, s_{j}\right\rangle_{h^{k}} \Omega
$$

for $\left(s_{i}\right)$ a basis of holomorphic sections of $H^{0}\left(L^{k}\right)$ with respect to $\mathrm{Hilb}(h)$. Then

$$
\left\|\mu_{\Omega}\left(h_{k}\right)-I_{\Omega, k}\right\|_{\text {op }} \rightarrow 0 \quad \text { as } k \rightarrow \infty,
$$

and the convergence is uniform for $\omega$ in a compact subset of Kähler metrics in $2 \pi c_{1}(L)$.

Proof. Firstly, the matrix $I_{\Omega, k}$ does not depend on the choice of the orthonormal basis $\left(s_{i}\right)_{i=1}^{N}$. Thanks to the asymptotic expansion given by Theorem 2.1,

$$
\mu_{\Omega}\left(h_{k}\right)=\int_{M}\left\langle s_{i}, s_{j}\right\rangle_{\mathrm{FS}(\operatorname{Hilb}(h))} \Omega=\int_{M}\left\langle s_{i}, s_{j}\right\rangle_{h^{k}}(1+O(1 / k)) \Omega .
$$

Finally, we can deduce the convergence by using [Don01, Lemma 28] which ensures that for the operator norm,

$$
\left\|\int_{M}\left\langle s_{i}, s_{j}\right\rangle_{\mathrm{FS}(\operatorname{Hilb}(h))} \times O(1 / k) \Omega\right\|_{\text {op }} \leq\left|\frac{\Omega}{\omega^{n}} O(1 / k)\right|_{L^{\infty}} .
$$

The uniformity of the convergence is given by the uniformity of the expansion in the asymptotics (see Theorem 2.1).

Given a tangent vector $A \in T_{b} \mathcal{B}$ with $b \in \mathcal{B}$, we have a vector field $\zeta_{A}$ on $\mathbb{P}^{N \vee}$ and thus on $M$, corresponding to $A$. Of course, the fact that $\mu_{\Omega}$ is a moment map (see Section 1) gives the following fact directly.

Lemma 4.2. For any pair of hermitian matrices $A, B \in T_{b} \mathcal{B}$, one has

$$
\operatorname{tr}\left(B d \mu_{\Omega}(A)\right)=\int_{M}\left(\zeta_{A}, \zeta_{B}\right) \Omega,
$$

where $(\cdot, \cdot)$ denotes the Fubini-Study inner product induced on the tangent vectors.

Lemma 4.3 ([Fin10, Lemma 18]). Let $A, B \in T_{b} \mathcal{B}$. Pointwise over $\mathbb{P}^{N \vee}$,

$$
H_{A} H_{B}+\left(\zeta_{A}, \zeta_{B}\right)=\operatorname{tr}(A B \mu) \text {. }
$$

Lemma 4.4. For any hermitian matrices $A, B \in T_{b} \mathcal{B}$,

$$
\operatorname{tr}\left(B d \mu_{\Omega}(A)\right)+\left\langle H_{A}, H_{B}\right\rangle_{L^{2}(M, \Omega)}=\operatorname{tr}\left(A B \mu_{\Omega}\right) .
$$


Proof. We start from the previous lemma which says that at each point of $M$,

$$
H_{A} H_{B}+\left(\zeta_{A}, \zeta_{B}\right)=\operatorname{tr}(A B \mu)
$$

Now, we integrate with respect to the volume form $\Omega$ and apply Lemma 4.2.

Lemma 4.5. For any hermitian matrix $A \in T_{b} \mathcal{B}$,

$$
\left\|H_{A}\right\|_{L^{2}(\Omega)}^{2} \leq\|A\|^{2}\left\|\mu_{\Omega}\right\|_{\text {op }}
$$

Proof. From the last lemma,

$$
\left\|H_{A}\right\|_{L^{2}(\Omega)}^{2}=\operatorname{tr}\left(A^{2} \mu_{\Omega}\right)-\operatorname{tr}\left(A d \mu_{\Omega}(A)\right) .
$$

Now, by Lemma 4.2,

$$
\operatorname{tr}\left(A d \mu_{\Omega}(A)\right)=\int_{M}\left(\zeta_{A}, \zeta_{A}\right) \Omega \geq 0
$$

Hence,

$$
\left\|H_{A}\right\|_{L^{2}(\Omega)}^{2} \leq \operatorname{tr}\left(A^{2} \mu_{\Omega}\right) \leq\|A\|^{2}\left\|\mu_{\Omega}\right\|_{\text {op }} .
$$

Lemma 4.6. For any hermitian matrix $A \in T_{b} \mathcal{B}$,

$$
\left\|d \mu_{\Omega}(A)\right\|_{\mathrm{op}} \leq\left\|d \mu_{\Omega}(A)\right\| \leq 2\|A\|\left\|\mu_{\Omega}\right\|_{\mathrm{op}} .
$$

Proof. From Lemma 4.4, one has

$$
\begin{aligned}
\left\|d \mu_{\Omega}(A)\right\|^{2} & =\operatorname{tr}\left(d \mu_{\Omega}(A)^{2}\right)=\operatorname{tr}\left(A d \mu_{\Omega}(A) \mu_{\Omega}\right)-\left\langle H_{A}, H_{d \mu_{\Omega}(A)}\right\rangle_{L^{2}(\Omega)} \\
& \leq\|A\|\left\|d \mu_{\Omega}(A)\right\|\left\|\mu_{\Omega}\right\|_{\text {op }}-\left\langle H_{A}, H_{d \mu_{\Omega}(A)}\right\rangle_{L^{2}(\Omega)} .
\end{aligned}
$$

Then we can conclude by using the fact that

$$
\left|\left\langle H_{A}, H_{d \mu_{\Omega}(A)}\right\rangle_{L^{2}(\Omega)}\right| \leq\left\|H_{A}\right\|_{L^{2}(\Omega)}\left\|H_{d \mu_{\Omega}(A)}\right\|_{L^{2}(\Omega)},
$$

and the previous lemma.

Finally, we obtain

Proposition 4.5. Let $b_{0}, b_{1} \in \mathcal{B}$. Then

$$
\left\|\mu_{\Omega}\left(b_{1}\right)\right\|_{\text {op }} \leq e^{2 \operatorname{dist}_{k}\left(b_{0}, b_{1}\right)}\left\|\mu_{\Omega}\left(b_{0}\right)\right\|_{\text {op }} .
$$

Proof. We know that a geodesic in the space $\mathcal{B}$ of Bergman metrics is given by a line, i.e., the hermitian metric involved is modified along the geodesic by $e^{t A}$ and that $\operatorname{dist}\left(b_{0}, b_{1}\right)$ $=\|A\|$. This can be rephrased by saying that if $\left(s_{i}^{0}\right)_{i=1}^{N+1}\left(\operatorname{resp} .\left(s_{i}^{1}\right)_{i=1}^{N+1}\right)$ is an orthonormal basis of $H^{0}\left(L^{k}\right)$ with respect to $b_{0}$ (resp. $\left.b_{1}\right)$, then there exists $\sigma \in \operatorname{GL}(N+1)$ such that $\sigma \cdot s^{0}=s^{1}$ and without loss of generality we can assume $\sigma$ is diagonal with entries $e^{\lambda_{0}}, \ldots, e^{\lambda_{n}}$. Then the geodesic is just induced by the family of bases $\sigma^{t} \cdot s^{0}$ for $t \in[0,1]$. Now, we can conclude our proof by using Lemma 4.6 and the fact that the norm $\|\cdot\|_{\text {op }}$ on the space of matrices is controlled from above by the Hilbert-Schmidt norm $\|\cdot\|$. 
We are now ready to give the proof of Theorem 1, that is, to show the smooth convergence of Kähler metrics $\omega_{k}(t)$ involved in the rescaled balancing flow (2) towards the solution $\omega_{t}$ to the $\Omega$-Kähler flow.

Proof of Theorem 1. Using Theorem 4, for any $m>0$, we have obtained a sequence of Kähler metrics

$$
\omega(k ; t)=c_{1}\left(h_{0} e^{\psi(k, t)}\right)
$$

such that $\omega(k ; t)$ converges, as $k \rightarrow \infty$ and in smooth sense, towards the solution $\omega_{t}=$ $c_{1}\left(h_{0} e^{\phi_{t}}\right)$ to the $\Omega$-Kähler flow. Moreover, for $k$ large enough and with $\bar{h}_{k}(t) \in \mathcal{B}$ the Bergman metric associated to $h_{0} e^{\psi(k, t)} \in \operatorname{Met}(L)$, one has the estimate

$$
\operatorname{dist}_{k}\left(h_{k}(t), \bar{h}_{k}(t)\right) \leq C / k^{m+1},
$$

where $h_{k}(t)$ is the metric induced by the rescaled $\Omega$-balancing flow. Consequently, in order to get the $C^{0}$ convergence in $t$, all we need to show is that

$$
\left\|\omega_{k}(t)-c_{1}\left(\bar{h}_{k}(t)\right)\right\|_{C^{r}\left(\omega_{t}\right)} \rightarrow 0
$$

The idea is to consider the geodesic in the Bergman space between these two points.

Firstly, we will see that along the geodesic from $\bar{h}_{k}(t)$ to $h_{k}(t)$ in $\mathcal{B},\left\|\mu_{\Omega}\right\|_{\text {op }}$ is controlled uniformly if we can apply Proposition 4.5. This requires proving that $\bar{h}_{k}(t)$ is within a uniformly bounded distance of $h_{k}(t)$ and that $\left\|\mu_{\Omega}\left(\bar{h}_{k}(t)\right)\right\|_{\text {op }}$ is bounded in $k$. But this comes from the fact that one can choose precisely $m \geq n+1$ in (26) and apply Proposition 4.4. For the latter, one needs to notice the estimate

$$
\left\|I_{\Omega, k}\right\|_{\text {op }} \leq \sup _{M} \frac{\Omega}{\omega^{n}}
$$

from [Don01, Lemma 28].

Secondly, we show that the points along this geodesic have $R$-bounded geometry. This is a consequence of Corollary 4.1, applied with the reference metric $\omega_{t}$ to the sequence $c_{1}\left(\bar{h}_{k}(t)\right)$. On one hand, $\left\|\mu_{\Omega}\left(\bar{h}_{k}(t)\right)\right\|_{\text {op }}$ is under control as we have just seen. On the other hand, $c_{1}\left(\bar{h}_{k}(t)\right)$ converge to $\omega_{t}$ in $C^{\infty}$ (hence in $C^{r+4}$ topology), thus they have $R / 2$ bounded geometry. Given $m \geq n+2$, one infers, thanks to Corollary 4.1 and inequality (26), that all the metrics along the geodesic from $\bar{h}_{k}(t)$ to $h_{k}(t)$ have $R$-bounded geometry in $C^{r+2}$.

Thirdly, we are exactly under the conditions of Lemma 4.1. It gives, by renormalizing the metrics in the Kähler class $2 \pi c_{1}(L)$ and by (26),

$$
\begin{aligned}
\left\|k \omega_{k}(t)-k c_{1}\left(\bar{h}_{k}(t)\right)\right\|_{C^{r}\left(k \omega_{t}\right)} & \leq C\left\|\mu_{\Omega}\left(\bar{h}_{k}(t)\right)\right\|_{\mathrm{op}} k^{n+2} \operatorname{dist}_{k}\left(h_{k}(t), \bar{h}_{k}(t)\right), \\
\left\|\omega_{k}(t)-c_{1}\left(\bar{h}_{k}(t)\right)\right\|_{C^{r}\left(\omega_{t}\right)} & \leq C\left\|\mu_{\Omega}\left(\bar{h}_{k}(t)\right)\right\|_{\mathrm{op}} k^{n+2-m-1+r / 2},
\end{aligned}
$$

where we have used the fact that the geodesic path from 0 to 1 is just a line. Here $C>0$ is a constant that does not depend on $k$. If we choose $m>r / 2+1+n$, we get the desired convergence in $C^{r}$ topology, i.e. (27). Of course, this reasoning yields uniform $C^{0}$ 
convergence in $t$ for $t \in \mathbb{R}_{+}$, because all the Kähler metrics $\omega_{t}$ that we are using are uniformly equivalent (we have convergence of the $\Omega$-Kähler flow, Theorem 3.2) and because we have the uniformity of the expansion in Theorems 2.1 and 2.2.

We now prove that one has $C^{1}$ convergence in $t$ of the flows $\omega_{k}(t)$. Again, we need to show the $C^{1}$ convergence of $\omega_{k}(t)$ to $c_{1}\left(\bar{h}_{k}(t)\right)$, because we already know the convergence of $c_{1}\left(\bar{h}_{k}(t)\right)$ to $\omega_{t}$ by Proposition 2.3. We are under the conditions of Lemma 4.1 by what we have just proved above. So we have, using again that our path is a geodesic,

$$
\begin{aligned}
\left\|k \frac{\partial \omega_{k}(t)}{\partial t}-k \frac{\partial c_{1}\left(\bar{h}_{k}(t)\right)}{\partial t}\right\|_{C^{r}} \leq & C^{*}\left\|\mu_{\Omega}\left(\bar{h}_{k}(t)\right)\right\|_{\mathrm{op}} k^{n+2} \operatorname{dist}_{k}\left(\frac{\left.\partial h_{k}(t)\right)}{\partial t}, \frac{\partial \bar{h}_{k}(t)}{\partial t}\right) \\
& +C^{*}\left\|d \mu_{\Omega}\left(\bar{h}_{k}(t)\right)\right\|_{\mathrm{op}} k^{n+2} \operatorname{dist}_{k}\left(h_{k}(t), \bar{h}_{k}(t)\right) .
\end{aligned}
$$

Here the $C^{r}$ norm is computed with respect to $k \omega_{t}$. If we apply Lemma 4.6, Theorem 4 and Proposition 4.2, we can bound from above the RHS of the last inequality to get

$$
\begin{aligned}
\left\|\frac{\partial \omega_{k}(t)}{\partial t}-\frac{\partial c_{1}\left(\bar{h}_{k}(t)\right)}{\partial t}\right\|_{C^{r}\left(\omega_{t}\right)} \leq & C^{\prime}\left\|\mu_{\Omega}\left(\bar{h}_{k}(t)\right)\right\|_{\mathrm{op}} k^{n+2-m-r / 2} \\
& +C^{\prime \prime}\left\|\mu_{\Omega}\left(\bar{h}_{k}(t)\right)\right\|_{\mathrm{op}} k^{n+2+r / 2} k^{-m-1} k^{-m-1} \\
\leq & C^{\prime \prime \prime} k^{n+2-m-r / 2} .
\end{aligned}
$$

Finally, we choose $m>r / 2+n+2$ to obtain $C^{1}$ convergence. This completes the proof of Theorem 1.

Finally, if we apply Definition 1.1 that asserts that an $\Omega$-balanced metric does always exist and is a zero of the moment map $\mu_{\Omega}^{0}$, Theorem 1 , and the convergence of the $\Omega$-Kähler flow to a solution to the Calabi problem, we directly obtain the following result.

Corollary 4.2. Under the same setting as above, the sequence of balanced metrics $h_{k}(\infty)^{1 / k} \in \operatorname{Met}(L)$, obtained as the limit of the balancing flow at $t=\infty$, converges in smooth topology to $h_{\infty}$, a solution of the Calabi problem,

$$
\left(c_{1}\left(h_{\infty}\right)\right)^{n}=\Omega .
$$

Note that this is a new proof of Theorem 1.1, but which uses a priori the existence of a solution to the Calabi problem (compare with [Kel09]).

\section{The infinite-dimensional set-up and generalizations}

\subsection{A symplectic approach to the Calabi problem}

In this section we develop the moment map set-up on the infinite-dimensional space of Kähler potentials related to the $\Omega$-Kähler flow. Assume that $(M, L)$ is a polarized manifold. Fix $\omega \in 2 \pi c_{1}(L)$ and $\Omega$ a smooth volume form on $M$ with $\int_{M} \Omega=\operatorname{Vol}_{L}(M)$. We introduce $\mathcal{M}$, the infinite-dimensional space of integrable hermitian connections on $L$ with Kähler form as curvature, with respect to a fixed complex structure. This means that 
we consider unitary connections $\nabla$ on $L$ such that if $F_{\nabla} \in \Omega^{2}(M$, $\operatorname{End}(L))$ is the curvature connection, then $F_{\nabla}^{0,2}=F_{\nabla}^{2,0}=0$ and $F_{\nabla}^{1,1}$ is a positive form with respect to the complex structure on $M$.

Consider the abelian gauge group $\mathcal{G}$ of maps $L \rightarrow L$ that cover the identity on $M$. By duality, the Lie algebra $\operatorname{Lie}(\mathcal{G})$ can be identified with the space of smooth functions from $M$ to $\mathbb{R}$ with zero integral, since one can identify $\mathcal{G}$ with $C^{\infty}\left(M, S^{1}\right)$. The tangent space at $\mathcal{M}$ is given by the 1 -forms with values in $\operatorname{End}(L)$. For simplicity, we assume that $M$ is simply connected and we fix the following symplectic form on $\mathcal{M}$ at the point $\nabla \in \mathcal{M}$ :

$$
\nu_{\nabla}(a, b)=\int_{M} a \wedge b \wedge F_{\nabla}^{n-1},
$$

which is a symplectic form invariant under the action of $\mathcal{G}$.

We have a natural paring $\operatorname{Lie}(\mathcal{G}) \times \operatorname{Lie}(\mathcal{G})^{*} \rightarrow \mathbb{R}$ given by

$$
(\zeta, \theta) \mapsto \int_{M} \zeta \theta=\int_{M}\langle\zeta, \theta\rangle
$$

We are in a moment map setting. Actually, we have the following simple proposition that shows that prescribing the volume form in a Kähler class is related to finding the zero of a certain moment map. Note that given $\nabla \in \mathcal{M}$, we let $A_{\nabla}$ be the real connection $\left(S^{1}\right.$ invariant) 1 -form associated to $\nabla$ on the natural $S^{1}$-principal bundle $\pi: P \rightarrow M$ that we can associate with $L \rightarrow M$. It acts on an element $\zeta \in \operatorname{Lie}(\mathcal{G})$ by decomposing it into a vertical and horizontal parts and fibrewise this vertical part corresponds to a rotation which is eventually parametrized by the real function $\left\langle A_{\nabla}, \zeta\right\rangle$ over $M$.

Proposition 5.1. There is a moment map $\bar{\mu}: \mathcal{M} \rightarrow \operatorname{Lie}(\mathcal{G})^{*}$ associated to the action of $\mathcal{G}$ on $(\mathcal{M}, v)$ given by

$$
\bar{\mu}(\nabla)=\left\langle A_{\nabla}, \cdot\right\rangle\left(\left(F_{\nabla}\right)^{n}-\Omega\right) .
$$

Proof. We need to check that for any $\zeta \in \operatorname{Lie}(\mathcal{G})$ and any vector field $V$, we have

$$
\langle d \bar{\mu}(\nabla)(V), \zeta\rangle=v_{\nabla}\left(V, X_{\zeta}\right)
$$

where $X_{\zeta}$ is the vector field on $\mathcal{M}$ defined by the infinitesimal action of $\zeta \in \operatorname{Lie}(\mathcal{G})$. More explicitly, $X_{\zeta}$ is given by $X_{\zeta}=L_{\zeta} A_{\nabla}=d\left\langle A_{\nabla}, \zeta\right\rangle+\iota_{\zeta} d A=d\left\langle A_{\nabla}, \zeta\right\rangle+\iota_{\pi_{*} \zeta} F_{\nabla}=$ $d\left\langle A_{\nabla}, \zeta\right\rangle$, since the elements of $\mathcal{G}$ cover the identity on $M$. Now, we have

$$
\nu_{\nabla}\left(V, X_{\zeta}\right)=\int_{M} V \wedge d\left\langle A_{\nabla}, \zeta\right\rangle \wedge F_{\nabla}^{n-1}=\int_{M}\left\langle A_{\nabla}, \zeta\right\rangle d V \wedge F_{\nabla}^{n-1} .
$$

But the change in $F_{\nabla}$ by the vector field $V$ is precisely given by $d V$, so

$$
\langle d \bar{\mu}(\nabla)(V), \zeta\rangle=\int_{X}\left\langle A_{\nabla}, \zeta\right\rangle d V \wedge F_{\nabla}^{n-1},
$$

since the elements of $\mathcal{G}$ cover the identity on $M$. 
Note that the moment map that we have just defined is obviously not unique. As in the proof above, denote by $X_{\zeta}$ the vector field associated to $\zeta \in \operatorname{Lie}(\mathcal{G})$. Now, using the pairing (28) and the natural $\mathcal{G}$-invariant norm on $\operatorname{Lie}(\mathcal{G})$, one can consider $\bar{\mu}$ with values in $\operatorname{Lie}(\mathcal{G})$, which means that we write $\bar{\mu}(\nabla)=\left(F_{\nabla}^{n}-\Omega\right) / F_{\nabla}^{n}$. Then we consider the gradient flow

$$
\frac{d}{d t}\left\|\bar{\mu}\left(\nabla_{t}\right)\right\|^{2}=-\left\|X_{\bar{\mu}\left(\nabla_{t}\right)}\right\|^{2}
$$

where the norm on the RHS is computed with respect to $v_{\nabla_{t}}$. This is actually equivalent to

$$
\frac{d A_{\nabla_{t}}}{d t}=I X_{\bar{\mu}\left(\nabla_{t}\right)}
$$

with $I$ the complex multiplication on the tangent vectors in $\mathcal{M}$. This equation can be rephrased in terms of flow over 1-forms by

$$
\frac{d F_{\nabla_{t}}}{d t}=L_{I \bar{\mu}\left(f_{t}\right)} F_{\nabla_{t}}
$$

If we use the notations of the previous sections, where $\omega_{t}=F_{\nabla_{t}}$ is an evolving Kähler form, then this (negative) gradient flow reads

$$
\frac{d \omega_{t}}{d t}=\sqrt{-1} \partial \bar{\partial}\left(\frac{\omega_{t}^{n}-\Omega}{\omega_{t}^{n}}\right) .
$$

Then, using the fact that the kernel of the operator $\sqrt{-1} \partial \bar{\partial}$ is given by constants (since $M$ is compact), one recovers precisely the equation of the $\Omega$-Kähler flow (9). Finally, we would like to mention that J. Fine [Fin11] has developed a more general theory that covers the results presented in this section (see [Fin11, Section 3.2]).

\subsection{Integral of a moment map}

In this section we deal with a very general set-up. Consider the case of a Kähler manifold ( $\Xi, \omega)$ polarized by the line bundle $L$ and a moment map $\mu$ associated to the action of a linear reductive group $\Gamma$ whose complexification acts holomorphically. To the moment map $\mu$ corresponds canonically a functional

$$
\Psi: \Xi \times \Gamma^{\mathbb{C}} \rightarrow \mathbb{R}
$$

that we call the integral of the moment map $\mu$ and that has the following two properties:

- For all $p \in \Xi$, the critical points of the restriction $\Psi_{p}$ of $\Psi$ to $\{p\} \times \Gamma^{\mathbb{C}}$ coincide with the points of the orbit $\operatorname{Orb}_{\Gamma \mathbb{C}}(p)$ on which the moment map vanishes;

- the restriction $\Psi_{p}$ to the "lines" $\left\{e^{\lambda u}: u \in \mathbb{R}\right\}$, where $\lambda \in \operatorname{Lie}\left(\Gamma^{\mathbb{C}}\right)$, is convex.

This is well known in the projective case from the seminal work of G. Kempf and L. Ness [KN79]. We also refer to [MiR00] for a more general setting.

Theorem 5.1. There exists a unique map $\Psi: \Xi \times \Gamma^{\mathbb{C}} \rightarrow \mathbb{R}$ that satisfies:

1. $\Psi(p, e)=0$ for all $p \in \Xi$;

2. $\left.\frac{d}{d u} \Psi\left(p, e^{i \lambda u}\right)\right|_{u=0}=\langle\mu(p), \lambda\rangle$ for all $\lambda \in \operatorname{Lie}(\Gamma)$. 
Let us sum up some of the main properties of the integral of the moment map.

Proposition 5.2. The functional $\Psi$ is $\Gamma$-invariant (for the left action) and satisfies the cocyclicity relation

$$
\Psi(p, \gamma)+\Psi\left(\gamma p, \gamma^{\prime}\right)=\Psi\left(p, \gamma^{\prime} \gamma\right)
$$

for all $p \in \Xi, \gamma, \gamma^{\prime} \in \Gamma^{\mathbb{C}}$, and the equivariance relation

$$
\Psi\left(\gamma p, \gamma^{\prime}\right)=\Psi\left(p, \gamma^{-1} \gamma^{\prime} \gamma\right)
$$

for all $p \in \Xi, \gamma \in \Gamma, \gamma^{\prime} \in \Gamma^{\mathbb{C}}$.

Moreover, $\frac{d^{2}}{d u^{2}} \Psi\left(p, e^{i \lambda u}\right) \geq 0$ for all $\lambda \in \operatorname{Lie}(\Gamma)$, with equality if and only if $X_{\lambda}\left(e^{i \lambda u} p\right)=0$.

Let us apply the previous results in our set-up. We introduce some classical functionals on the space of Kähler potentials. The energy functionals $I, J$, introduced by T. Aubin [Aub84] (see also [Tia00]), are defined for each pair $\left(\omega, \omega_{\phi}:=\omega+\sqrt{-1} \partial \bar{\partial} \phi\right)$ by

$$
\begin{aligned}
& I\left(\omega, \omega_{\phi}\right)=\frac{1}{V} \int_{M} \sqrt{-1} \partial \phi \wedge \bar{\partial} \phi \wedge \sum_{i=0}^{n-1} \omega^{i} \wedge \omega_{\phi}^{n-1-i}=\frac{1}{V} \int_{M} \phi\left(\omega^{n}-\omega_{\phi}^{n}\right), \\
& J\left(\omega, \omega_{\phi}\right)=\frac{1}{V(n+1)} \int_{M} \sqrt{-1} \partial \phi \wedge \bar{\partial} \phi \wedge \sum_{i=0}^{n-1}(n-i) \omega^{i} \wedge \omega_{\phi}^{n-1-i},
\end{aligned}
$$

where we have skipped again the normalization of the volume form by the factor $n$ ! for simplicity. Note that

$$
J\left(\omega, \omega_{\phi}\right)=\int_{0}^{1} \frac{I(\omega, \omega+s \sqrt{-1} \partial \bar{\partial} \phi)}{s} d s .
$$

It is well known that $I, J$ and $I-J$ are all nonnegative and equivalent. One may also define these functionals via a variational formula, and they are very natural from this point of view. We refer to the recent work of [BBGZ09] where this idea is exploited in detail. If $\omega_{\phi_{t}}$ is a smooth path in the Kähler cone, a direct computation gives

$$
\frac{d}{d t} J\left(\omega, \omega_{\phi_{t}}\right)=\frac{1}{V} \int_{M} \dot{\phi}_{t}\left(\omega^{n}-\omega_{\phi_{t}}^{n}\right) .
$$

We obtain

Proposition 5.3. The integral of the moment map associated to $\bar{\mu}: \mathcal{M} \rightarrow \operatorname{Lie}(\mathcal{G})^{*}$ is given by the functional

$$
F_{\Omega}^{0}\left(\omega, \omega_{\phi}\right)=J\left(\omega, \omega_{\phi}\right)+\frac{1}{V} \int_{M} \phi\left(\Omega-\omega^{n}\right) .
$$

In particular, this functional is decreasing along the $\Omega$-Kähler flow. This can be checked directly since, along the flow (9), one has

$$
\frac{d}{d t} F_{\Omega}^{0}\left(\omega, \omega_{\phi_{t}}\right)=\int_{M} \dot{\phi}_{t}\left(\Omega-\omega_{\phi_{t}}^{n}\right)=-\int_{M} \frac{\left(\omega_{\phi_{t}}^{n}-\Omega\right)^{2}}{\omega_{\phi_{t}}^{n}} \leq 0 .
$$


On the other hand, for the second derivative along the $\Omega$-Kähler flow, one gets

$$
\frac{d^{2}}{d t^{2}} F_{\Omega}^{0}\left(\omega, \omega_{\phi_{t}}\right)=\int_{M} \frac{\dot{\phi}_{t} \ddot{\phi}_{t}\left(-2+\dot{\phi}_{t}\right)}{\left(1-\dot{\phi}_{t}\right)^{2}} \Omega
$$

by using the fact that $\frac{d \omega_{\phi_{t}}^{n}}{d t}=\frac{\ddot{\phi}_{t}}{\left(1-\dot{\phi}_{t}\right)^{2}} \Omega$. The term $-2+\dot{\phi}_{t}$ is always negative from (9), since $\Omega / \omega_{\phi_{t}}^{n}$ is positive. We can apply the maximum principle to check that the derivative of $\left(\dot{\phi}_{t}\right)^{2}$ is negative. So the right hand side of (30) is actually positive and the functional $t \mapsto F_{\Omega}^{0}\left(\omega, \omega_{\phi_{t}}\right)$ is convex along the $\Omega$-Kähler flow. Finally, it is clear that this functional has the cocyclicity property

$$
F_{\Omega}^{0}\left(\omega, \omega_{\phi_{1}}\right)=F_{\Omega}^{0}\left(\omega, \omega_{\phi_{2}}\right)+F_{\Omega}^{0}\left(\omega_{\phi_{2}}, \omega_{\phi_{1}}\right) .
$$

\subsection{The degenerate cases}

One can ask if the main results of this paper hold at least partially when one considers nonample classes or degenerate volume forms. Let us explain which arguments used in the previous sections can be extended with no major difficulty to show the existence of the balancing flow and its convergence as $k \rightarrow \infty$ for nonsmooth volume forms.

First of all, a careful reading of the proof of Proposition 2.1 shows that the asymptotic expansion of the Bergman function holds when one considers a positive volume form $\Omega$ that can be written as

$$
\Omega=f_{\Omega} \omega^{n}
$$

with $f_{\Omega}>0$ on $M$ and $f_{\Omega} \in L_{\omega}^{1}(M, \mathbb{R})$ and $\omega$ a smooth Kähler form. Then, the asymptotic result for the operator $Q_{k}$ (Theorem 2.2) is valid when applied to the space of functions $f \in L_{\omega}^{p}(M, \mathbb{R})$ with $p>1$. This comes from the techniques of [LM07] that can be extended from $L^{2}$ to $L^{p}$ topology, $p>1$. To be more precise, the regularity of the function $f$ is only needed in [LM07, (27)], and the Cauchy-Schwarz inequality in [LM07, (28)] can still be applied in $L^{p}$ spaces. This implies that we get a more general version of Theorem 3 for $\Omega \in L^{p}(M)$ a positive volume form $(p>1)$ but with a weaker underlying convergence (the error terms are only controlled in $L^{p}$ norms instead of $C^{\infty}$ norm for the sequences $\omega_{k}(t)$ and $\left.\partial \omega_{k}(t) / \partial t\right)$. Finally, when one considers nonsmooth forms $\Omega=f_{\Omega} \omega^{n}$ with $f_{\Omega} \in L_{\omega}^{p}(M), f_{\Omega}>0$ with $p>1$, the limit of the balancing flows is still the $\Omega$-Kähler flow (3). Note that we do not expect the potential of the metric involved in (3) to be smooth and we shall speak instead of weak $\Omega$-Kähler flow. We also remark that the balancing flow will converge to a balanced metric again. Actually a notion of balanced metric for $L^{p}$ volume forms (and even more general) has been studied in detail in the recent work [BBGZ09, Section 7]. Furthermore the technical results for Section 4 still hold. Thus, we are able to derive an analogue of Theorem 1 as soon as we have an existence and convergence result for the weak $\Omega$-Kähler flow in the infinite-dimensional set-up.

Moreover we expect that this method can be generalized with no major difficulty to $L^{p}$ semi-positive forms, i.e. $f_{\Omega} \geq 0$. This will be studied in a forthcoming paper using the techniques developed by S. Kołodziej in his generalization of the Calabi problem [Kol98]. 
Finally, if one considers $L$ to be a big line bundle and if one assumes the existence of a semi-positive smooth closed real $(1,1)$-form $\omega_{0} \in c_{1}(L)$, the results of R. Berman [Ber09, Theorems 1.1, 1.2] can be applied to derive a version of Proposition 2.1. In that case, the equilibrium metric corresponds to $\omega_{0}$ and one has the asymptotic expansion (5) in the sense of measures. In this set-up, we believe that what is happening on the space of Kähler potentials for the $\Omega$-Kähler flow is related to the very general version of the Calabi problem studied by the viscosity method à la P.-L. Lions in [EGZ11, EGZ09]. In particular we expect the following conjecture to hold.

Conjecture 5.1. Assume that $L$ is a big line bundle, and $\Omega>0$ a volume form with $L^{p}$ density, $p>1$, such that $\int_{M} \omega_{0}^{n}=\int_{M} \Omega$. Then the $\Omega$-Kähler flow exists in a weak sense and converges to a solution of the Calabi problem

$$
\left(\omega+\sqrt{-1} \partial \bar{\partial} \phi_{\infty}\right)^{n}=\Omega
$$

with $\phi_{\infty} \in C^{0}(M), \phi_{\infty} \in C^{1,1}(K)$ for any compact subset $K \subset M \backslash \mathbb{B}_{+}(L)$ where $\mathbb{B}_{+}(L)$ is the augmented base locus of $L$, which is an analytic subvariety of $M$.

Acknowledgments. The research of the first author is partially supported by NSF grant DMS0909581. He would like to thank Xiaofeng Sun for helpful conversations. The second author is very grateful to J. Fine for enlightening conversations about Section 5.1. He also wishes to thank deeply R. Berman, S. Boucksom, Z. Błocki, V. Guedj and P. Eyssidieux. Both authors are grateful to the referee whose insightful comments improved the preliminary version a lot.

\section{References}

[Aub84] Aubin, T.: Réduction du cas positif de l'équation de Monge-Ampère sur les variétés kählériennes compactes à la démonstration d'une inégalité. J. Funct. Anal. 57, 143-153 (1984) Zbl 0538.53063 MR 0749521

[Bak11] Baker, C.: The mean curvature flow of submanifolds of high codimension. PhD Thesis, arXiv:1104.4409v1 (2011)

[Ber09] Berman, R. J.: Bergman kernels and equilibrium measures for line bundles over projective manifolds. Amer. J. Math. 131, 1485-1524 (2009) Zbl 1191.32008 MR 2559862

[BBGZ09] Berman, R., Boucksom, S., Guedj, V., Zeriahi, A.: A variational approach to complex Monge-Ampère equations. arXiv:0907.4490 (2009)

[Bou90] Bouche, T.: Convergence de la métrique de Fubini-Study d'un fibré linéaire positif. Ann. Inst. Fourier (Grenoble) 40, 117-130 (1990) Zbl 0685.32015 MR 1056777

[Cao85] Cao, H. D.: Deformation of Kähler metrics to Kähler-Einstein metrics on compact Kähler manifolds. Invent. Math. 81, 359-372 (1985) Zbl 0574.53042 MR 0799272

[Cat99] Catlin, D.: The Bergman kernel and a theorem of Tian. In: Analysis and Geometry in Several Complex Variables (Katata, 1997), Trends Math., Birkhäuser, 1-23 (1999) Zbl 0941.32002 MR 1699887

[Don01] Donaldson, S. K.: Scalar curvature and projective embeddings. I. J. Differential Geom. 59, 479-522 (2001) Zbl 1052.32017 MR 1916953

[Don09] Donaldson, S. K.: Some numerical results in complex differential geometry. Pure Appl. Math. Quart. 5, 571-618 (2009) Zbl 1178.32018 MR 2508897

[EGZ09] Eyssidieux, P., Guedj, V., Zeriahi, A.: Singular Kähler-Einstein metrics. J. Amer. Math. Soc. 22, 607-639 (2009) Zbl 1215.32017 MR 2505296 
[EGZ11] Eyssidieux, P., Guedj, V., Zeriahi, A.: Viscosity solutions to complex MongeAmpère equations. Comm. Pure Appl. Math. 64, 1059-1094 (2011) Zbl 1227.32042 MR 2839271

[FLM11] Fang, H., Lai, M., Ma, X.: On a class of fully nonlinear flows in Kähler geometry. J. Reine Angew. Math. 653, 189-220 (2011) Zbl 1222.53070 MR 2794631

[Fin10] Fine, J.: Calabi flow and projective embeddings. J. Differential Geom. 84, 489-523 (2010) Zbl 1202.32018 MR 2669363

[Fin11] Fine, J.: The hamiltonian geometry of the space of unitary connections with symplectic curvature. arXiv:1101.2420v1 (2011)

[GT01] Gilbarg, D., Trudinger, N. S.: Elliptic Partial Differential Equations of Second Order. Classics in Math., Springer, Berlin (2001) (reprint of the 1998 edition) Zbl 1042.35002 MR 1814364

[Kel09] Keller, J.: Ricci iterations on Kähler classes. J. Inst. Math. Jussieu 8, 743-768 (2009) Zbl 1178.32020 MR 2540879

[KN79] Kempf, G., Ness, L.: The length of vectors in representation spaces. In: Algebraic Geometry (Copenhagen, 1978), Lecture Notes in Math. 732, Springer, Berlin, 233-243 (1979) Zbl 0407.22012 MR 0555701

[Ko198] Kołodziej, S.: The complex Monge-Ampère equation. Acta Math. 180, 69-117 (1998) Zbl 0913.35043 MR 1618325

[LY86] Li, P., Yau, S.-T.: On the parabolic kernel of the Schrödinger operator. Acta Math. 156, 153-201 (1986) Zbl 0611.58045 MR 0834612

[LM07] Liu, K., Ma, X.: A remark on: "Some numerical results in complex differential geometry" [arxiv.org/abs/math/0512625] by S. K. Donaldson. Math. Res. Lett. 14, 165-171 (2007) Zbl 1175.32014 MR 2318616

[Lu00] Lu, Z.: On the lower order terms of the asymptotic expansion of Tian-Yau-Zelditch. Amer. J. Math. 122, 235-273 (2000) Zbl 0972.53042 MR 1749048

[MM07] Ma, X., Marinescu, G.: Holomorphic Morse Inequalities and Bergman Kernels, Progr. Math.. 254, Birkhäuser, Basel (2007) Zbl 1135.32001 MR 2339952

[MM10] Ma, X., Marinescu, G.: Berezin-Toeplitz quantization on Kähler manifolds. J. Reine Angew. Math. 662, 1-56 (2012); addendum, ibid., 57-58 Zbl 1251.47030 MR 2876259

[MiR00] Mundet i Riera, I.: A Hitchin-Kobayashi correspondence for Kähler fibrations. J. Reine Angew. Math. 528, 41-80 (2000) Zbl 1002.53057 MR 1801657

[Rua98] Ruan, W.-D.: Canonical coordinates and Bergmann metrics. Comm. Anal. Geom. 6, 589-631 (1998) Zbl 0917.53026 MR 1638878

[Tia90] Tian, G.: On a set of polarized Kähler metrics on algebraic manifolds. J. Differential Geom. 32, 99-130 (1990) Zbl 0706.53036 MR 1064867

[Tia00] Tian, G.: Canonical Metrics in Kähler Geometry. Lectures in Math. ETH Zürich, Birkhäuser, Basel (2000) Z Zbl 0978.53002 MR 1787650

[Yau78] Yau, S.-T.: On the Ricci curvature of a compact Kähler manifold and the complex Monge-Ampère equation. I. Comm. Pure Appl. Math. 31, 339-411 (1978) Zbl 0369.53059 MR 0480350

[Yau86] Yau, S.-T.: Nonlinear analysis in geometry. Enseign. Math. 33, 109-158 (1986) Zbl 0631.53003 MR 0865650

[Ze198] Zelditch, S.: Asymptotics of holomorphic sections of powers of a positive line bundle. In: Séminaire sur les Équations aux Dérivées Partielles, 1997-1998, exp. XXII, École Polytech., Palaiseau, 1998, 12 pp. Z Zbl 1057.32501 MR 1660535 